\title{
Synthesis and Analgesic Activity of New Heterocyclic Cyanothioacetamide Derivatives
}

\author{
I. V. Bibik ${ }^{a}$, E. Yu. Bibik ${ }^{b}$, V. V. Dotsenko ${ }^{b, c, *}$, K. A. Frolov ${ }^{b}$, S. G. Krivokolysko ${ }^{b}$, N. A. Aksenov , $^{c}$ \\ I. V. Aksenova ${ }^{c}$, S. V. Shcherbakov ${ }^{c}$, and S. N. Ovcharov ${ }^{c}$ \\ ${ }^{a}$ Belgorod State National Research University, Belgorod, 308015 Russia \\ ${ }^{b}$ Kuban State University, Krasnodar, 350040 Russia \\ ${ }^{c}$ North Caucasus Federal University, Stavropol, 355009 Russia \\ *e-mail: victor_dotsenko_@mail.ru
}

Received December 16, 2020; revised December 16, 2020; accepted December 28, 2020

\begin{abstract}
The reaction of cyanothioacetamide with aromatic aldehydes and 1,3-dicarbonyl compounds followed by aminomethylation or $S$-alkylation gave a series of heterocyclic derivatives with a 1,2,3,4-tetrahydropyridine or 1,4,5,6,7,8-hexahydroquinoline fragment. The resulting compounds were tested for analgesic activity in vivo. Some of the prepared compounds showed an antinociceptive effect superior to that of ketorolac in dynamics.
\end{abstract}

Keywords: cyanothioacetamide, Mannich reaction, pyrido[2,1-b][1,3,5]thiadiazines, 2-oxo-3-cyano-1,4,5,6tetrahydropyridine-2-thiolates, analgesics

DOI: $10.1134 / \mathrm{S} 107036322102002 \mathrm{X}$

Cyanothioacetamide $\mathbf{1}$ is a readily available and multifunctional reagent widely used in synthetic organic chemistry [1-4]. One of the main directions of the use of thioamide 1 is the preparation of a wide range of $S, N$ heterocyclic compounds, the most important of which are 3-cyanopyridine-2(1H)-thiones 2 [5-11] (Scheme 1). Compounds 2 are a convenient scaffolds for the synthesis of a wide range of thieno[2,3- $b]$ pyridines [12-20], thiazolo[3,2-a]pyridines [21-29], pyrido[2,1-b][1,3,5]thiadiazines [30-34], dipyrido[1,2-a:1'2'-e][1,3,5,7]tetrazocines [35], pyrido[1,2-a][1,3,5] triazines [36], isothiazolo[5,4-b]pyridines [37-40], and other bi- and polycyclic structures.

Many of the compounds obtained in this way exhibit valuable practical properties. In particular, pyrido[2,1-b]$[1,3,5]$ thiadiazine derivatives 3 (Scheme 2) show high activity against tick-borne encephalitis virus and Powassan virus [41], exhibit a pronounced antiinflammatory effect [42], analeptic [43] and adaptogenic effect [44]. Disulfides 4 (Hlg = F, Br) have moderate antiHIV activity [45], compounds $\mathbf{5}$ and $\mathbf{6}$ are inhibitors of autotaxin [46], while hexahydroquinoline 7 inhibits betaamyloid peptide formation [47], which is considered one of the main factors in the development of the Alzheimer's disease.

The broad spectrum of biological activity found in partially saturated nicotinonitrile derivatives readily available from $\alpha$-cyanothioacetamide stimulates further research in the search for new biologically active substances in this series.

The aim of this work was to synthesize new structural analogs of compounds 3-7 and study their analgesic activity in vivo (rats). Cyanothioacetamide derivatives were selected for our research based on in silico assessment of bioavailability parameters and potential targets using open software packages OSIRIS Property Explorer [48], SwissADME [49], SwissTargetPrediction [50], Molinspiration Property Calculation Service [51], and admetSAR [52]. Thus, we selected 6 new compounds from a small (250+ compounds) library of structural analogs of $\mathbf{3}-\mathbf{7}$, namely pyrido[2,1-b][1,3,5]thiadiazines $\mathbf{8 a}-\mathbf{8 c}, \alpha$-(hetarylthio)acetanilides $\mathbf{9 , 1 0}$, and hexahydroquinoline 11 (Scheme 3).

The syntheses of compounds $\mathbf{8}-\mathbf{1 1}$ are depicted in Schemes 4-6. Thus, pyrido[2,1-b][1,3,5]thiadiazines 8a-8c (Scheme 4) were prepared in 4 steps, starting 
Scheme 1.<smiles>[R]c1nc2sc(F)c(N)c2c([R])c1[R]</smiles>

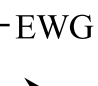<smiles>[R]C1=C([R])N2C=CSC2=C(C#N)C([R])C1CCC</smiles><smiles>[R]c1nc2snc(Br)c2c([R])c1[R]</smiles><smiles>[R]c1c(C#N)c2n(c(=O)c1[R])CN([R])CS2</smiles><smiles>[R]N1CSC2=C(C#N)C([Al])CC(=O)N2C1</smiles>

3<smiles>COc1cc(C2C(C#N)=C(SCC(=O)Nc3cccc4ccccc34)NC3=C2C(=O)CC(C)(C)C3)ccc1O</smiles>

6<smiles></smiles>

Scheme 2.

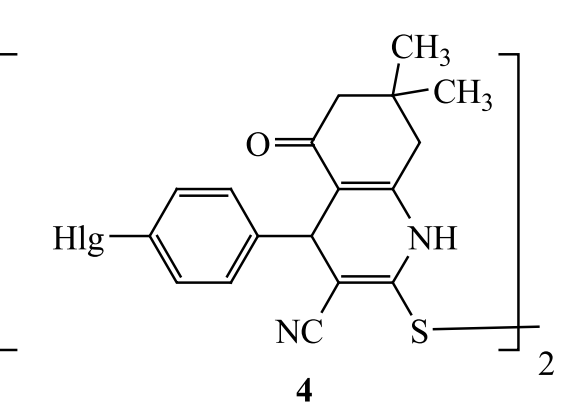<smiles>COc1cc(C2CC(=O)NC(SCC(=O)Nc3ccc(Br)cc3)=C2C#N)ccc1O</smiles>

from cyanothioacetamide $\mathbf{1}$. The reaction of thioamide 1 with 3-methoxy-4-ethoxybenzaldehyde afforded thioacrylamide 12, which reacted without isolation with Meldrum acid 13 to form the corresponding Michael<smiles>Cc1c(Cl)cccc1NC(=O)CSC1=C(C#N)C(c2cccs2)C2=C(CC(C)(C)CC2=O)N1</smiles>

7 
Scheme 3.<smiles>CCOc1ccc(C2CC(=O)N3CN(c4ccc(CC)cc4)CSC3=C2C#N)cc1OC</smiles><smiles>CCOc1ccc(C2CC(=O)N3CN(c4ccc(OC)cc4OC)CSC3=C2C#N)cc1OC</smiles>

$8 c$<smiles>CCOC(=O)C1C(=O)NC(SCC(=O)Nc2ccccc2CC)=C(C#N)C1c1ccccc1F</smiles>

10

in the presence of an excess of formaldehyde under mild conditions resulted in the formation of the target pyrido[2,1- $b][1,3,5]$ thiadiazines $\mathbf{8 a - 8 c}$ in high yields.

Compound 9 was synthesized by reacting cyanothioacetamide 1 with cyclohexane-1,3-dione and vanillin in three steps similar to the known procedure with intermediate isolation of mercaptoquinoline 16 [53] (Scheme 5). Hexahydroquinoline 11 was obtained by a modified method described in [54]. Finally, tetrahydropyridine $\mathbf{1 0}$ was prepared similar to procedure reported in $[55,56]$ (Scheme 6).

Compounds 8a-8c, 9-11 are fine crystalline substances of white or beige color, poorly soluble in ethanol and diethyl ether, soluble in acetone and DMSO. Their structure was confirmed by a complex of ${ }^{1} \mathrm{H},{ }^{13} \mathrm{C}$ DEPTQ NMR and IR spectral data, as well as high-resolution mass spectrometry method. In addition, structure of compounds $\mathbf{8 c}$ and 9 was proved using two-dimensional NMR spectroscopy methods $\left({ }^{1} \mathrm{H}-{ }^{13} \mathrm{C} \mathrm{HSQC},{ }^{1} \mathrm{H}-{ }^{13} \mathrm{C} \mathrm{HMBC}\right)$ (Tables 1,2). The complete set of observed correlations<smiles>CCOc1ccc(C2CC(=O)N3CN(c4ccc(C)c(C)c4)CSC3=C2C#N)cc1OC</smiles>

8b<smiles>COc1cc(C2C(C#N)=C(SCC(=O)Nc3ccc(F)cc3)NC3=C2C(=O)CCC3)ccc1O</smiles><smiles>COc1ccc(C2C(C#N)=C(SC)NC3=C2C(=O)CC(C)(C)C3)cc1OC</smiles>

11 and signal assignments are presented in Supplementary Information.

Analgesic activity of compounds $\mathbf{8}-\mathbf{1 1}$ was studied in vivo using the well-known thermal tail immersion test [57-60], as well as classical formalin-induced orofacial trigeminal pain test [61-65]. The average time of the tail withdrawal from a vessel with hot water in rats without pharmacological correction is $3.57 \mathrm{~s}$ (Table 3). Preliminary administration of ketorolac to rats of the reference group significantly increased this value to $10.92 \mathrm{~s}$.

In was found that hexahydroquinolines $9(10.15 \mathrm{~s})$ and 11 (10.90 s) show the most pronounced analgesic activity. Pyrido[2,1- $b][1,3,5]$ thiadiazines $\mathbf{8 a}-\mathbf{8 c}$ have moderate analgesic activity and increase more than two times the tail flick time in the heat immersion test. In contrast, pyridine derivative $\mathbf{1 0}$ does not exhibit analgesic properties.

Data on analgesic activity in the orofacial trigeminal pain test are presented in Table 4 . Thus, in 10 min we 
Scheme 4.

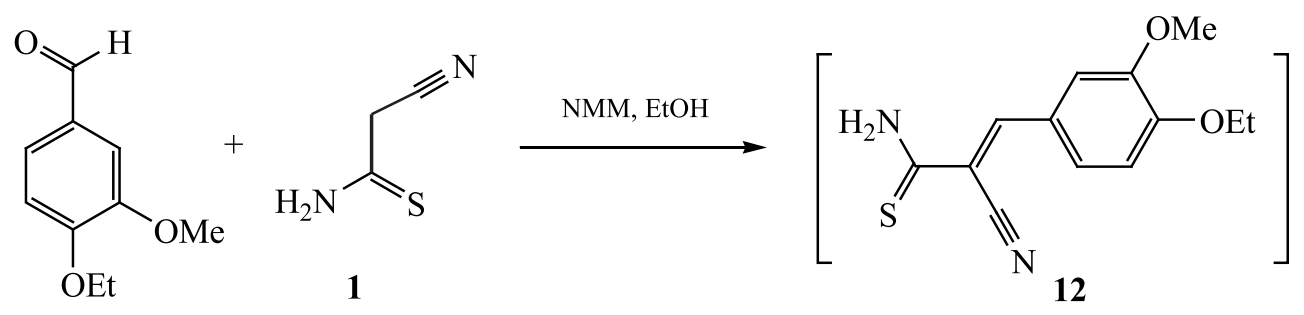

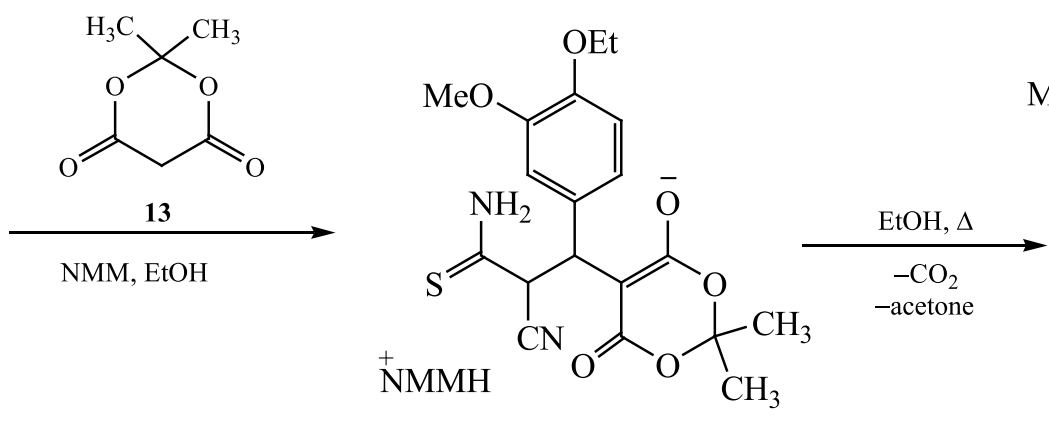

14<smiles>[R]N1CSC2=C(C#N)C(c3ccc(OCC)c(OC)c3)CC(=O)N2C1</smiles>

15

\section{$\mathbf{8 a}-\mathbf{8 c}$}

$\mathrm{NMM}=N$-methylmorpholine; $8 \mathrm{R}=4-\mathrm{EtC}_{6} \mathrm{H}_{4}(\mathbf{a}) ; 3,4-\mathrm{Me}_{2} \mathrm{C}_{6} \mathrm{H}_{3}(\mathbf{b}) ; 2,4-(\mathrm{MeO})_{2} \mathrm{C}_{6} \mathrm{H}_{3}$ (c) .

recorded an average of 173 scratching movements of the orofacial area in rats of the control group. In $15 \mathrm{~min}$ this value was 250 , and in $20 \mathrm{~min}-294$ movements. Preliminary administration of ketorolac to rats of the reference group decreased the frequency of scratching movements by $41.04 \%$ in $10 \mathrm{~min}$ in comparison with the control (Table 4). A 15-min time interval after the introduction of algogen into the vibrissa region in rats of this group showed a decrease in this value by $46.6 \%$. Within $20 \mathrm{~min}$, the frequency of scratching movements decreased by $43.23 \%$ compared with the control.

Compounds 9 and 11 showed the highest analgesic activity in the orofacial trigeminal pain test already in the first 10 min, while compounds $8 \mathbf{a}$ and $\mathbf{b}$ showed effect comparable to the reference drug (ketorolac). Within a 15-min interval after the administration of algogen, the leader in analgesic activity was compound 11, which was $19.3 \%$ more effective than ketorolac. Hexahydroquinoline derivative 9 showed an activity exceeding that of ketorolac by $14.66 \%$. Compound $\mathbf{8 b}$ exhibited activity comparable to ketorolac. Finally, within a 20 -min interval after the injection of formalin solution into the vibrissa area, the maximum analgesic activity was revealed for hexahydroquinolines 9 and $\mathbf{1 1}$. They exceed the reference drug in analgesic activity by 24.4 and $16.5 \%$, respectively. The analgesic activity of compound $\mathbf{8 b}$ also slightly exceeds that of ketorolac.

In the orofacial trigeminal pain test, pyridothiadiazine derivative 8c showed low analgesic activity; we did not find significant differences in comparison with the control group of animals. Compound $\mathbf{1 0}$ at a similar dose did not show any analgesic effect, since at none of the 
Scheme 5.<smiles>COc1cc(C=O)ccc1O</smiles><smiles>COc1cc(C2C(C#N)=C(S)NC3=C2C(=O)CCC3)ccc1O</smiles>

16<smiles>N#CCC(N)=S</smiles>

1

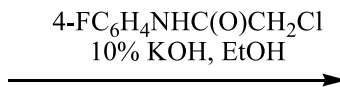

\section{Scheme 5.}


Table 1. Correlations in the ${ }^{1} \mathrm{H}-{ }^{13} \mathrm{C}$ HSQC and ${ }^{1} \mathrm{H}-{ }^{13} \mathrm{C}$ HMBC NMR spectra of compound 8c

\begin{tabular}{|c|c|c|}
\hline \multirow{2}{*}{$\delta_{\mathrm{H}}, \mathrm{ppm}$} & \multicolumn{2}{|r|}{$\delta_{\mathrm{C}}, \mathrm{ppm}$} \\
\hline & ${ }^{1} \mathrm{H}-{ }^{13} \mathrm{C}$ HSQC & ${ }^{1} \mathrm{H}-{ }^{13} \mathrm{C} \mathrm{HMBC}$ \\
\hline $1.30 \mathrm{t}\left(3 \mathrm{H}, \mathrm{OCH}_{2} \mathrm{C}_{3}\right)$ & $14.8 *\left(\mathrm{CH}_{3} \mathrm{CH}_{2} \mathrm{O}\right)$ & $63.6\left(\mathrm{CH}_{3} \mathrm{CH}_{2} \mathrm{O}\right)$ \\
\hline 2.69 d. d $\left(1 \mathrm{H}\right.$, cis $\left.-\mathrm{C}^{7} \mathrm{H}\right)$ & $38.2\left(\mathrm{C}^{7} \mathrm{H}_{2}\right)$ & 37.3* $\left(\mathrm{C}^{8} \mathrm{H}\right), 88.7\left(\mathrm{C}^{9}\right), 132.1\left(\mathrm{C}^{1} \mathrm{C}-\mathrm{Ar}\right), 167.3(\mathrm{C}=\mathrm{O})$ \\
\hline 3.02 d. d $\left(1 \mathrm{H}\right.$, trans $\left.-\mathrm{C}^{7} \mathrm{H}\right)$ & $38.2\left(\mathrm{C}^{7} \mathrm{H}_{2}\right)$ & 37.3* $\left(\mathrm{C}^{8} \mathrm{H}\right), 88.7\left(\mathrm{C}^{9}\right), 132.1\left(\mathrm{C}^{1} \mathrm{C}-\mathrm{Ar}\right), 167.3(\mathrm{C}=\mathrm{O})$ \\
\hline $3.71 \mathrm{~s}(3 \mathrm{H}, \mathrm{MeO})$ & $55.3 *\left(\mathrm{CH}_{3} \mathrm{O} \mathrm{C}-\mathrm{Ar}\right)$ & $149.0\left(\mathrm{C}^{3}-\mathrm{OMe} \mathrm{C}-\mathrm{Ar}\right)$ \\
\hline $3.74 \mathrm{~s}(3 \mathrm{H}, \mathrm{MeO})$ & $55.4 *\left(4-\mathrm{CH}_{3} \mathrm{O} \mathrm{N}-\mathrm{Ar}\right)$ & $157.1\left(\mathrm{C}^{4}-\mathrm{OMe} \mathrm{N}-\mathrm{Ar}\right)$ \\
\hline $3.79 \mathrm{~s}(3 \mathrm{H}, \mathrm{MeO})$ & $55.7 *\left(2-\mathrm{CH}_{3} \mathrm{O} \mathrm{N}-\mathrm{Ar}\right)$ & $152.8\left(\mathrm{C}^{2}-\mathrm{OMe} \mathrm{N}-\mathrm{Ar}\right)$ \\
\hline $3.83-3.85 \mathrm{~m}\left(1 \mathrm{H}, \mathrm{C}^{8} \mathrm{H}\right)$ & $37.3^{*}\left(\mathrm{C}^{8} \mathrm{H}\right)$ & $\begin{array}{l}38.2\left(\mathrm{C}^{7} \mathrm{H}_{2}\right), 88.7\left(\mathrm{C}^{9}\right), 111.0^{*}\left(\mathrm{C}^{2} \mathrm{H} \mathrm{C}-\mathrm{Ar}\right), 117.9(\mathrm{C} \equiv \mathrm{N}), \\
132.1\left(\mathrm{C}^{1} \mathrm{C}-\mathrm{Ar}\right), 149.3\left(\mathrm{C}^{9 \mathrm{a}}\right), 167.3(\mathrm{C}=\mathrm{O}) .\end{array}$ \\
\hline $3.96 \mathrm{q}\left(\mathrm{OC}_{2} \mathrm{CH}_{3}\right)$ & $63.6\left(\mathrm{CH}_{3} \underline{\mathrm{CH}}_{2} \mathrm{O}\right)$ & $14.8^{*}\left(\mathrm{C}_{3} \mathrm{CH}_{2} \mathrm{O}\right), 147.3\left(\mathrm{C}^{4}-\mathrm{OEt} \mathrm{C}-\mathrm{Ar}\right)$ \\
\hline $5.10-5.28 \mathrm{~m}\left(4 \mathrm{H}, \mathrm{NCH}_{2} \mathrm{NCH}_{2} \mathrm{~S}\right)$ & $54.7\left(\mathrm{C}^{2} \mathrm{H}_{2}\right), 60.4\left(\mathrm{C}^{4} \mathrm{H}_{2}\right)$ & $\begin{array}{l}54.7\left(\mathrm{C}^{2} \mathrm{H}_{2}\right), 60.4\left(\mathrm{C}^{4} \mathrm{H}_{2}\right), 127.3\left(\mathrm{C}^{1} \mathrm{~N}-\mathrm{Ar}\right), 149.3\left(\mathrm{C}^{9 \mathrm{a}}\right) \\
167.3(\mathrm{C}=\mathrm{O})\end{array}$ \\
\hline $\begin{array}{l}6.42-6.48 \mathrm{~m}\left(2 \mathrm{H}, \mathrm{H}^{5} \mathrm{~N}-\mathrm{Ar}, \mathrm{H}^{6}\right. \\
\text { C-Ar) }\end{array}$ & $\begin{array}{l}104.2^{*}\left(\mathrm{C}^{5} \mathrm{H} \mathrm{N}-\mathrm{Ar}, 118.2 *\right. \\
\left(\mathrm{C}^{6} \mathrm{H} \mathrm{C}-\mathrm{Ar}\right)\end{array}$ & $\begin{array}{l}37.3^{*}\left(\mathrm{C}^{8} \mathrm{H}\right), 100.0^{*}\left(\mathrm{C}^{3} \mathrm{H} \text { N-Ar }\right), 111.0^{*}\left(\mathrm{C}^{2} \mathrm{H} \mathrm{C}-\mathrm{Ar}\right), 127.3 \\
\left(\mathrm{C}^{1} \mathrm{~N}-\mathrm{Ar}\right), 147.3\left(\mathrm{C}^{4}-\mathrm{OEt} \mathrm{C}-\mathrm{Ar}\right), 157.1\left(\mathrm{C}^{4}-\mathrm{OMe} \mathrm{N}-\mathrm{Ar}\right)\end{array}$ \\
\hline $6.60 \mathrm{~d}\left(1 \mathrm{H}, \mathrm{H}^{3} \mathrm{~N}-\mathrm{Ar}\right)$ & $100.0^{*}\left(\mathrm{C}^{3} \mathrm{H} \mathrm{N}-\mathrm{Ar}\right)$ & $\begin{array}{l}\left.\text { 104.2* ( } \mathrm{C}^{5} \mathrm{H} \text { N-Ar }\right), 127.3\left(\mathrm{C}^{1} \mathrm{~N}-\mathrm{Ar}\right), 152.8\left(\mathrm{C}^{2}-\mathrm{OMe}\right. \\
\mathrm{N}-\mathrm{Ar}), 157.1\left(\mathrm{C}^{4}-\mathrm{OMe} \mathrm{N}-\mathrm{Ar}\right)\end{array}$ \\
\hline $6.75 \mathrm{~d}\left(1 \mathrm{H}, \mathrm{H}^{5} \mathrm{C}-\mathrm{Ar}\right)$ & $112.6^{*}\left(\mathrm{C}^{5} \mathrm{H} \mathrm{C}-\mathrm{Ar}\right)$ & $132.1\left(\mathrm{C}^{1} \mathrm{C}-\mathrm{Ar}\right), 149.0\left(\mathrm{C}^{3}-\mathrm{OMe} \mathrm{C}-\mathrm{Ar}\right)$ \\
\hline $6.79 \mathrm{~d}\left(1 \mathrm{H}, \mathrm{H}^{2} \mathrm{C}-\mathrm{Ar}\right)$ & $111.0 *\left(\mathrm{C}^{2} \mathrm{H} \mathrm{C}-\mathrm{Ar}\right)$ & $\begin{array}{l}37.3^{*}\left(\mathrm{C}^{8} \mathrm{H}\right), 118.2^{*}\left(\mathrm{C}^{6} \mathrm{H} \mathrm{C}-\mathrm{Ar}\right), 147.3\left(\mathrm{C}^{4}-\mathrm{OEt} \mathrm{C}-\mathrm{Ar}\right) \\
149.0\left(\mathrm{C}^{3}-\mathrm{OMe} \mathrm{C}-\mathrm{Ar}\right)\end{array}$ \\
\hline $7.01 \mathrm{~d}\left(1 \mathrm{H}, \mathrm{H}^{6} \mathrm{~N}-\mathrm{Ar}\right)$ & $121.0 *\left(\mathrm{C}^{6} \mathrm{H} \mathrm{N}-\mathrm{Ar}\right)$ & $\begin{array}{l}127.3\left(\mathrm{C}^{1} \mathrm{~N}-\mathrm{Ar}\right), 152.8\left(\mathrm{C}^{2}-\mathrm{OMe} \mathrm{N}-\mathrm{Ar}\right), 157.1\left(\mathrm{C}^{4}-\mathrm{OMe}\right. \\
\mathrm{N}-\mathrm{Ar})\end{array}$ \\
\hline
\end{tabular}

containing tetrahydropyridine or hexahydroquinoline fragments. The biological screening showed the presence of the most pronounced analgesic activity at a dose of 5 $\mathrm{mg} / \mathrm{kg}$ exceeding that of ketorolac in two compounds, namely 2-\{[4-(4-hydroxy-3-methoxyphenyl)-5-oxo3-cyano-1,4,5,6,7,8-hexahydroquinolin-2-yl]thio $N$-(4-fluorophenyl)acetamide and 7,7-dimethyl-2methylthio-4-(3,4-dimethoxyphenyl)-5-oxo-1,4,5,6,7,8hexahydroquinoline-3-carbonitrile. In addition, pyrido$[2,1-b][1,3,5]$ thiadiazine derivatives showed analgesic activity comparable to that of ketorolac.

\section{EXPERIMENTAL}

IR spectra were registered on a Bruker Vertex 70 spectrometer equipped with an ATR sample accessory. NMR spectra were recorded on a Bruker Avance III HD $400 \mathrm{MHz}$ instrument $\left[400.17\left({ }^{1} \mathrm{H}\right), 100.63 \mathrm{MHz}\left({ }^{13} \mathrm{C}\right)\right]$ using DMSO- $d_{6}$ as a solvent; residual solvent signals were used as a standard. High-resolution mass spectra (HRMS) were recorded on a Bruker maXis Impact quadrupole TOF mass spectrometer using a $\mathrm{MeCN}$-water system, $\mathrm{HCO}_{2} \mathrm{Na}-\mathrm{HCO}_{2} \mathrm{H}$ calibration, and ESI-TOF ionization. Individuality of the obtained compounds was monitored by TLC on Silufol UV-254 plates, eluting with acetonehexane mixture $(1: 1)$ and developing with iodine vapor or UV light.

Cyanothioacetamide 1 [66] and Meldrum's acid 13 [67] were prepared according to the known methods.

General procedure for the synthesis of 3-aryl8-(3-methoxy4-ethoxyphenyl)-6-oxo- $2,3,4,6,7,8$ hexahydropyrido $[2,1-b][1,3,5]$ thiadiazine-9-carbonitriles 8a-8c. $N$-Methylmorpholine (2 drops) was added with stirring to a mixture of 3-methoxy-4-ethoxybenzaldehyde (1.8 g, $10 \mathrm{mmol})$ and cyanothioacetamide $1(1.0,10 \mathrm{mmol})$ in $15 \mathrm{~mL}$ of ethanol. The resulting mixture was stirred until complete conversion, while the formation of a yellow-orange precipitate of the 
Table 2. Correlations in the ${ }^{1} \mathrm{H}-{ }^{13} \mathrm{C}$ HSQC and ${ }^{1} \mathrm{H}-{ }^{13} \mathrm{C}$ HMBC NMR spectra of compound 9

\begin{tabular}{|c|c|c|}
\hline \multirow{2}{*}{$\delta_{\mathrm{H}}, \mathrm{ppm}$} & \multicolumn{2}{|r|}{$\delta_{\mathrm{C}}, \mathrm{ppm}$} \\
\hline & ${ }^{1} \mathrm{H}-{ }^{13} \mathrm{C}$ HSQC & ${ }^{1} \mathrm{H}-{ }^{13} \mathrm{C}$ HMBC \\
\hline $1.75-1.93 \mathrm{~m}\left(2 \mathrm{H}, \mathrm{C}^{7} \mathrm{H}_{2}\right)$ & $20.7\left(\mathrm{C}^{7} \mathrm{H}_{2}\right)$ & $151.3\left(\mathrm{C}^{8 \mathrm{a}}\right), 194.9(\mathrm{C}=\mathrm{O})$ \\
\hline $2.21-2.25 \mathrm{~m}\left(2 \mathrm{H}, \mathrm{C}^{6} \mathrm{H}_{2}\right)$ & $36.7\left(\mathrm{C}^{6} \mathrm{H}_{2}\right)$ & $20.7\left(\mathrm{C}^{7} \mathrm{H}_{2}\right), 26.3\left(\mathrm{C}^{8} \mathrm{H}_{2}\right), 194.9(\mathrm{C}=\mathrm{O})$ \\
\hline $2.49-2.59 \mathrm{~m}\left(2 \mathrm{H}, \mathrm{C}^{8} \mathrm{H}_{2}\right)$ & $26.3\left(\mathrm{C}^{8} \mathrm{H}_{2}\right)$ & $20.7\left(\mathrm{C}^{7} \mathrm{H}_{2}\right), 109.2\left(\mathrm{C}^{4 \mathrm{a}}\right), 151.3\left(\mathrm{C}^{8 \mathrm{a}}\right)$ \\
\hline $3.70 \mathrm{~s}(3 \mathrm{H}, \mathrm{MeO})$ & $55.5 *(\mathrm{OMe})$ & $147.4\left(\mathrm{C}^{5}-\mathrm{OMe} \mathrm{Ar}\right)$ \\
\hline $3.90 \mathrm{q}\left(2 \mathrm{H}, \mathrm{SCH}_{2}\right)$ & $36.8\left(\mathrm{SCH}_{2}\right)$ & $142.1\left(\mathrm{C}^{2}\right), 166.6(\mathrm{C}(\mathrm{O}) \mathrm{NH})$ \\
\hline $4.40 \mathrm{~s}\left(1 \mathrm{H}, \mathrm{H}^{4}\right)$ & $38.6^{*}\left(\mathrm{C}^{4} \mathrm{H}\right)$ & $\begin{array}{l}92.0\left(\mathrm{C}^{3}\right), 109.2\left(\mathrm{C}^{4 a}\right), 111.5^{*}\left(\mathrm{C}^{2} \mathrm{H} \mathrm{Ar}\right), 119.4^{*}\left(\mathrm{C}^{6} \mathrm{H} \mathrm{Ar}\right), \\
136.1\left(\mathrm{C}^{1} \mathrm{Ar}\right), 142.1\left(\mathrm{C}^{2}\right), 151.3\left(\mathrm{C}^{8 \mathrm{a}}\right), 194.9(\mathrm{C}=\mathrm{O})\end{array}$ \\
\hline 6.53 d. d $\left(1 \mathrm{H}, \mathrm{H}^{6} \mathrm{Ar}\right)$ & $119.4 *\left(\mathrm{C}^{6} \mathrm{H} \mathrm{Ar}\right)$ & $38.6^{*}\left(\mathrm{C}^{4} \mathrm{H}\right), 111.5^{*}\left(\mathrm{C}^{2} \mathrm{H} \mathrm{Ar}\right), 145.5\left(\mathrm{C}^{4}-\mathrm{OH} \mathrm{Ar}\right)$ \\
\hline $6.64 \mathrm{~d}\left(1 \mathrm{H}, \mathrm{H}^{5} \mathrm{Ar}\right)$ & $115.4 *\left(\mathrm{C}^{5} \mathrm{H} \mathrm{Ar}\right)$ & $136.1\left(\mathrm{C}^{1} \mathrm{Ar}\right), 145.5\left(\mathrm{C}^{4}-\mathrm{OH} \mathrm{Ar}\right), 147.4\left(\mathrm{C}^{5}-\mathrm{OMe} \mathrm{Ar}\right)$ \\
\hline $6.70 \mathrm{~d}\left(1 \mathrm{H}, \mathrm{H}^{2} \mathrm{Ar}\right)$ & $111.5^{*}\left(\mathrm{C}^{2} \mathrm{H} \mathrm{Ar}\right)$ & $\begin{array}{l}38.6^{*}\left(\mathrm{C}^{4} \mathrm{H}\right), 119.4^{*}\left(\mathrm{C}^{6} \mathrm{H} \mathrm{Ar}\right), 136.1\left(\mathrm{C}^{1} \mathrm{Ar}\right), 145.5\left(\mathrm{C}^{4}-\mathrm{OH}\right. \\
\mathrm{Ar}), 147.4\left(\mathrm{C}^{5}-\mathrm{OMe} \mathrm{Ar}\right)\end{array}$ \\
\hline $\begin{array}{l}7.14-7.18 \mathrm{~m}\left(2 \mathrm{H}, \mathrm{H}^{3}, \mathrm{H}^{5}\right. \\
\left.4-\mathrm{FC}_{6} \mathrm{H}_{4}\right)\end{array}$ & $115.5^{*} \mathrm{~d}\left(\mathrm{C}^{3} \mathrm{H}, \mathrm{C}^{5} \mathrm{H} 4-\mathrm{FC}_{6} \mathrm{H}_{4}\right)$ & $\begin{array}{l}115.5^{*} \mathrm{~d}\left(\mathrm{C}^{3} \mathrm{H}, \mathrm{C}^{5} \mathrm{H} 4-\mathrm{FC}_{6} \mathrm{H}_{4}\right), 134.8 \mathrm{~d}\left(\mathrm{C}^{1} 4-\mathrm{FC}_{6} \mathrm{H}_{4}\right), 158.3 \mathrm{~d} \\
\left(\mathrm{C}^{4} 4-\mathrm{FC}_{6} \mathrm{H}_{4}\right)\end{array}$ \\
\hline $\begin{array}{l}7.52-7.56 \mathrm{~m}\left(2 \mathrm{H}, \mathrm{H}^{2}, \mathrm{H}^{6}\right. \\
\left.4-\mathrm{FC}_{6} \mathrm{H}_{4}\right)\end{array}$ & $121.4 * \mathrm{~d}\left(\mathrm{C}^{2} \mathrm{H}, \mathrm{C}^{6} \mathrm{H} 4-\mathrm{FC}_{6} \mathrm{H}_{4}\right)$ & $\begin{array}{l}121.4 * \mathrm{~d}\left(\mathrm{C}^{2} \mathrm{H}, \mathrm{C}^{6} \mathrm{H} 4-\mathrm{FC}_{6} \mathrm{H}_{4}\right), 134.8 \mathrm{~d}\left(\mathrm{C}^{1} 4-\mathrm{FC}_{6} \mathrm{H}_{4}\right), 158.3 \mathrm{~d} \\
\left(\mathrm{C}^{4} 4-\mathrm{FC}_{6} \mathrm{H}_{4}\right)\end{array}$ \\
\hline $8.86 \mathrm{~s}(1 \mathrm{H}, \mathrm{OH})$ & - & $115.4 *\left(\mathrm{C}^{5} \mathrm{H} \mathrm{Ar}\right), 145.5\left(\mathrm{C}^{4}-\mathrm{OH} \mathrm{Ar}\right), 147.4\left(\mathrm{C}^{5}-\mathrm{OMe} \mathrm{Ar}\right)$ \\
\hline $9.99 \mathrm{~s}\left(1 \mathrm{H}, \mathrm{N}^{1} \mathrm{H}\right)$ & - & $26.3\left(\mathrm{C}^{8} \mathrm{H}_{2}\right), 92.0\left(\mathrm{C}^{3}\right), 109.2\left(\mathrm{C}^{4 \mathrm{a}}\right), 151.3\left(\mathrm{C}^{8 \mathrm{a}}\right)$ \\
\hline $10.39 \mathrm{~s}[1 \mathrm{H}, \mathrm{C}(\mathrm{O}) \mathrm{NH}]$ & - & $\begin{array}{l}121.4^{*} \mathrm{~d}\left(\mathrm{C}^{2} \mathrm{H}, \mathrm{C}^{6} \mathrm{H} 4-\mathrm{FC}_{6} \mathrm{H}_{4}\right), 134.8 \mathrm{~d}\left(\mathrm{C}^{1} 4-\mathrm{FC}_{6} \mathrm{H}_{4}\right), 166.6 \\
{[\mathrm{C}(\mathrm{O}) \mathrm{NH}]}\end{array}$ \\
\hline
\end{tabular}

Table 3. Analgesic activity of compounds 8-11 in the test of thermal immersion of the tail in rats

\begin{tabular}{l|c|c|c|c}
\hline \multirow{2}{*}{ Compound } & \multicolumn{4}{|c}{ Tail withdrawal time measurement results, $\mathrm{s}$} \\
\cline { 2 - 5 } & average value & standard deviation & median value & $p^{\text {a }}$ \\
\hline Control & 3.57 & 1.16 & 3.85 & - \\
Ketorolac & 10.92 & 2.36 & 10.10 & 0.03 \\
$\mathbf{8 a}$ & 8.68 & 2.79 & 8.45 & 0.03 \\
$\mathbf{8 b}$ & 9.20 & 4.74 & 10.00 & 0.03 \\
$\mathbf{8 c}$ & 7.60 & 2.65 & 7.50 & 0.03 \\
$\mathbf{9}$ & 10.15 & 2.67 & 10.75 & 0.03 \\
$\mathbf{1 0}$ & 3.52 & 1.61 & 3.40 & 0.92 \\
$\mathbf{1 1}$ & 10.90 & 3.96 & 8.95 & 0.03 \\
\hline
\end{tabular}

${ }^{a} p$-reliability of the results in comparison with the control group of animals.

Knoevenagel condensation product, 3-(3-methoxy-4ethoxyphenyl)-2-cyanothioacrylamide 12, was observed. To a suspension of thioacrylamide $\mathbf{1 2}$ were added $1.50 \mathrm{~g}$ (10.4 mmol) of Meldrum acid 13, $10 \mathrm{~mL}$ of ethanol and $1.65 \mathrm{~mL}(15 \mathrm{mmol})$ of $N$-methylmorpholine. The suspension was stirred until the reaction completed (control by TLC, color change from orange to white). A suspension of Michael adduct $\mathbf{1 4}$ was boiled with vigorous stirring until a clear yellow-orange solution was formed and then for another $30 \mathrm{~min}$ until the reaction was 
Table 4. Analgesic activity of compounds 8-11 in the test of orofacial trigeminal pain in rats

\begin{tabular}{l|c|c|r|c|c|c|c|c|c|c|c|c}
\hline \multirow{2}{*}{ Compound } & \multicolumn{4}{|c|}{10 min } & \multicolumn{4}{c|}{15 min } & \multicolumn{4}{c}{20 min } \\
& $\begin{array}{c}\text { average } \\
\text { value }\end{array}$ & $\begin{array}{c}\text { standard } \\
\text { deviation }\end{array}$ & $\begin{array}{c}\text { median } \\
\text { value }\end{array}$ & $p^{\text {a }}$ & $\begin{array}{c}\text { average } \\
\text { value }\end{array}$ & $\begin{array}{c}\text { standard } \\
\text { deviation }\end{array}$ & $\begin{array}{c}\text { median } \\
\text { value }\end{array}$ & $p^{\text {a }}$ & $\begin{array}{c}\text { average } \\
\text { value }\end{array}$ & $\begin{array}{c}\text { standard } \\
\text { deviation }\end{array}$ & $\begin{array}{c}\text { median } \\
\text { value }\end{array}$ & $p^{\text {a }}$ \\
\hline Control & 173.50 & 35.77 & 183.00 & - & 250.83 & 40.89 & 243.50 & - & 294.33 & 61.78 & 296.50 & - \\
Ketorolac & 102.33 & 20.78 & 98.50 & 0.03 & 133.50 & 34.05 & 124.50 & 0.03 & 166.33 & 45.31 & 167.00 & 0.03 \\
$\mathbf{8 a}$ & 121.00 & 40.77 & 102.00 & 0.17 & 156.00 & 55.43 & 131.50 & 0.04 & 199.50 & 59.78 & 184.00 & 0.03 \\
$\mathbf{8 b}$ & 103.17 & 24.60 & 99.50 & 0.03 & 133.83 & 26.92 & 135.00 & 0.03 & 161.67 & 30.50 & 161.00 & 0.03 \\
$\mathbf{8 c}$ & 152.67 & 33.24 & 151.50 & 0.46 & 219.67 & 84.08 & 196.00 & 0.46 & 276.33 & 106.59 & 262.00 & 0.60 \\
$\mathbf{9}$ & 84.00 & 24.71 & 87.00 & 0.03 & 113.50 & 30.68 & 120.50 & 0.03 & 138.67 & 23.69 & 144.50 & 0.03 \\
$\mathbf{1 0}$ & 173.67 & 26.25 & 172.00 & 0.75 & 240.67 & 32.57 & 239.50 & 0.69 & 287.50 & 31.69 & 290.00 & 0.83 \\
$\mathbf{1 1}$ & 87.50 & 27.04 & 89.00 & 0.03 & 107.33 & 23.63 & 104.50 & 0.03 & 125.50 & 28.59 & 116.50 & 0.03 \\
\hline
\end{tabular}

${ }^{a} p$-reliability of the results in comparison with the control group of animals.

complete. The hot reaction mixture was evaporated to $1 / 3$ of the volume in vacuum, while $N$-methylmorpholinium 4-(3-methoxy-4-ethoxyphenyl)-6-oxo-3-cyano1,4,5,6tetrahydropyridine-2-thiolate 15 crystallized. Yield $2.8 \mathrm{~g}$ (69\%), pale yellow crystals. Thiolate 15 was used without further purification. ${ }^{1} \mathrm{H}$ NMR spectrum, $\delta$, ppm: $1.29 \mathrm{t}$ $\left(3 \mathrm{H}, \mathrm{OCH}_{2} \mathrm{CH}_{3},{ }^{3} \mathrm{~J}=7.0 \mathrm{~Hz}\right), 2.35 \mathrm{~d}$. d $\left(1 \mathrm{H}\right.$, cis $-\mathrm{C}^{5} \mathrm{H}$, $\left.{ }^{2} J=16.1,{ }^{3} J=4.4 \mathrm{~Hz}\right), 2.66 \mathrm{~d}$. d $\left(1 \mathrm{H}\right.$, trans $-\mathrm{C}^{5} \mathrm{H},{ }^{2} J=$ $\left.16.1,{ }^{3} J=6.9 \mathrm{~Hz}\right), 2.78 \mathrm{~s}\left(3 \mathrm{H}, \mathrm{NCH}_{3}\right), 3.13-3.22 \mathrm{~m}(4 \mathrm{H}$, $\left.\mathrm{CH}_{2} \mathrm{NCH}_{2}\right), 3.56$ d. d $\left(1 \mathrm{H}, \mathrm{C}^{4} \mathrm{H},{ }^{3} J=6.9,{ }^{3} J=4.4 \mathrm{~Hz}\right)$, $3.71 \mathrm{~s}(3 \mathrm{H}, \mathrm{MeO}), 3.71-3.80 \mathrm{~m}\left(4 \mathrm{H}, \mathrm{CH}_{2} \mathrm{OCH}_{2}\right), 3.95 \mathrm{q}$ $\left(\mathrm{OCH}_{2} \mathrm{CH}_{3},{ }^{3} \mathrm{~J}=7.0 \mathrm{~Hz}\right), 6.64 \mathrm{~d}$. d $\left(1 \mathrm{H}, \mathrm{H}-\mathrm{Ar},{ }^{3} \mathrm{~J}=8.1\right.$, $\left.{ }^{4} J=1.6 \mathrm{~Hz}\right), 6.78 \mathrm{~d}\left(1 \mathrm{H}, \mathrm{H}-\mathrm{Ar},{ }^{4} J=1.6 \mathrm{~Hz}\right), 6.83 \mathrm{~d}(1 \mathrm{H}$, $\left.\mathrm{H}-\mathrm{Ar},{ }^{3} J=8.1 \mathrm{~Hz}\right), 8.55$ br. s $(1 \mathrm{H}, \mathrm{NH}), 9.61$ v. br. $\mathrm{s}$ $\left(1 \mathrm{H}, \mathrm{NH}^{+}\right)$.

Thiolate 15 (0.5 g, $1.2 \mathrm{mmol})$ was dissolved by heating in $10 \mathrm{~mL}$ of $70 \%$ ethanol and filtered through a paper filter to obtain a clear solution. To the resulting solution was added the corresponding substituted aniline $(1.2 \mathrm{mmol})$ and an excess of $37 \%$ aqueous $\mathrm{HCHO}(1 \mathrm{~mL}$, $13.3 \mathrm{mmol})$. The mixture was refluxed with vigorous stirring for 2-3 min until crystallization of the reaction product, then cooled to room temperature and kept for $6 \mathrm{~h}$. The precipitate was filtered off, washed with water, cooled ethanol, and diethyl ether. If necessary, it was purified by recrystallization from acetone.

8-(3-Methoxy-4-ethoxyphenyl)-3-(4-ethylphenyl)6-oxo- $2,3,4,6,7,8$-hexahydropyrido $[2,1-b][1,3,5]-$ thiadiazine-9-carbonitrile (8a). Yield 87\%, colorless crystals. IR spectrum, $v, \mathrm{~cm}^{-1}: 2193 \mathrm{~s}(\mathrm{C} \equiv \mathrm{N}), 1691 \mathrm{~s}$ $(\mathrm{C}=\mathrm{O}) .{ }^{1} \mathrm{H}$ NMR spectrum, $\delta, \mathrm{ppm}: 1.16 \mathrm{t}(3 \mathrm{H}$, $\left.\mathrm{ArCH}_{2} \mathrm{CH}_{3},{ }^{3} \mathrm{~J}=7.6 \mathrm{~Hz}\right), 1.30 \mathrm{t}\left(3 \mathrm{H}, \mathrm{OCH}_{2} \mathrm{CH}_{3},{ }^{3} \mathrm{~J}=\right.$ $7.0 \mathrm{~Hz}), 2.55 \mathrm{q}\left(\mathrm{ArCl}_{2} \mathrm{CH}_{3},{ }^{3} \mathrm{~J}=7.6 \mathrm{~Hz}\right), 2.70 \mathrm{~d} . \mathrm{d}(1 \mathrm{H}$, cis $\left.-\mathrm{C}^{7} \mathrm{H},{ }^{2} J=16.0,{ }^{3} \mathrm{~J}=5.1 \mathrm{~Hz}\right), 3.00 \mathrm{~d} . \mathrm{d}\left(1 \mathrm{H}\right.$, trans $-\mathrm{C}^{7} \mathrm{H}$, $\left.{ }^{2} J=16.0,{ }^{3} J=7.1 \mathrm{~Hz}\right), 3.69 \mathrm{~s}(3 \mathrm{H}, \mathrm{MeO}), 3.80 \mathrm{~d} . \mathrm{d}(1 \mathrm{H}$, $\left.\mathrm{C}^{8} \mathrm{H},{ }^{3} \mathrm{~J}=7.1,{ }^{3} \mathrm{~J}=5.1 \mathrm{~Hz}\right), 3.96 \mathrm{q}\left(2 \mathrm{H}, \mathrm{OCH}_{2} \mathrm{CH}_{3},{ }^{3} \mathrm{~J}=\right.$ $7.0 \mathrm{~Hz}), 5.33 \mathrm{~d}\left(1 \mathrm{H}, \mathrm{CH}_{2} \mathrm{~S},{ }^{2} J=12.5 \mathrm{~Hz}\right), 5.39-5.42 \mathrm{~m}$ $\left(3 \mathrm{H}, \mathrm{CH}_{2} \mathrm{NCH}_{2} \mathrm{~S}\right), 6.45$ d. d $\left(1 \mathrm{H}, \mathrm{H}-\mathrm{Ar},{ }^{3} \mathrm{~J}=8.3,{ }^{4} \mathrm{~J}=2.0\right.$ $\mathrm{Hz}), 6.73 \mathrm{~d}\left(1 \mathrm{H}, \mathrm{H}-\mathrm{Ar},{ }^{3} \mathrm{~J}=8.3 \mathrm{~Hz}\right), 6.78 \mathrm{~d}(1 \mathrm{H}, \mathrm{H}-\mathrm{Ar}$, $\left.{ }^{4} J=2.0 \mathrm{~Hz}\right), 7.00 \mathrm{~d}\left(2 \mathrm{H}, \mathrm{H}-\mathrm{Ar},{ }^{3} J=8.6 \mathrm{~Hz}\right), 7.16 \mathrm{~d}$ $\left(2 \mathrm{H}, \mathrm{H}-\mathrm{Ar},{ }^{3} \mathrm{~J}=8.6 \mathrm{~Hz}\right) .{ }^{13} \mathrm{C}$ DEPTQ NMR spectrum, $\delta_{\mathrm{C}}, \mathrm{ppm}: 14.8^{*}\left(\underline{\mathrm{CH}}_{3} \mathrm{CH}_{2} \mathrm{O}\right), 15.6^{*}\left(\underline{\mathrm{CH}}_{3} \mathrm{CH}_{2} \mathrm{Ar}\right), 27.2$ $\left(\mathrm{CH}_{3} \underline{\mathrm{CH}}_{2} \mathrm{Ar}\right), 37.4 *\left(\mathrm{C}^{8} \mathrm{H}\right), 38.1\left(\mathrm{C}^{7} \mathrm{H}_{2}\right), 52.6\left(\mathrm{C}^{2} \mathrm{H}_{2}\right)$, 55.4* $\left(\mathrm{CH}_{3} \mathrm{O}\right), 57.8\left(\mathrm{C}^{4} \mathrm{H}_{2}\right), 63.6\left(\mathrm{CH}_{3} \underline{\mathrm{CH}}_{2} \mathrm{O}\right), 89.3\left(\mathrm{C}^{9}\right)$, $111.1^{*}(\mathrm{CH} \mathrm{Ar}), 112.7^{*}(\mathrm{CH} \mathrm{Ar}), 116.5^{*}\left(2 \mathrm{C}, \mathrm{C}^{2}, \mathrm{C}^{6}\right.$ $\left.4-\mathrm{EtC}_{6} \mathrm{H}_{4}\right), 117.6(\mathrm{C} \equiv \mathrm{N}), 128.8^{*}\left(2 \mathrm{C}, \mathrm{C}^{3}, \mathrm{C}^{5} 4-\mathrm{EtC}_{6} \mathrm{H}_{4}\right)$, 131.9 (C Ar), 136.8 (C Ar), 141.5 (C Ar), 147.3 (C ${ }^{4}$-OEt C-Ar), $148.8\left(\mathrm{C}^{3}-\mathrm{OMe} \mathrm{C}-\mathrm{Ar}\right), 149.1\left(\mathrm{C}^{\mathrm{9a}}\right), 167.3(\mathrm{C}=\mathrm{O})$. Hereinafter, an asterisk denotes signals in antiphase. Mass spectrum (HRMS ESI-TOF), $m / z$ : $472.1663[M+$ $\mathrm{Na}]^{+}$(calcd. for $\mathrm{C}_{25} \mathrm{H}_{27} \mathrm{~N}_{3} \mathrm{NaO}_{3} \mathrm{~S}: 472.1665$ ), 540.1553 $[M+\mathrm{HCOONa}+\mathrm{Na}]^{+}$(calcd. for $\mathrm{C}_{26} \mathrm{H}_{28} \mathrm{~N}_{3} \mathrm{Na}_{2} \mathrm{O}_{5} \mathrm{~S}$ : $540.1545)$.

3-(3,4-Dimethylphenyl)-8-(3-methoxy-4-ethoxyphenyl)-6-oxo-2,3,4,6,7,8-hexahydropyrido $[2,1-b]-$ $[1,3,5]$ thiadiazine-9-carbonitrile $(8 b)$. Yield $87 \%$, colorless crystals. IR spectrum, $v, \mathrm{~cm}^{-1}: 2191 \mathrm{~s}(\mathrm{C} \equiv \mathrm{N})$, $1689 \mathrm{~s}(\mathrm{C}=\mathrm{O}) .{ }^{1} \mathrm{H}$ NMR spectrum, $\delta$, ppm: $1.30 \mathrm{t}(3 \mathrm{H}$, $\left.\mathrm{OCH}_{2} \mathrm{CH}_{3},{ }^{3} \mathrm{~J}=7.0 \mathrm{~Hz}\right), 2.16 \mathrm{~s}(3 \mathrm{H}, \mathrm{Me}-\mathrm{Ar}), 2.17 \mathrm{~s}$ $(3 \mathrm{H}, \mathrm{Me}-\mathrm{Ar}), 2.70 \mathrm{~d}$. d $\left(1 \mathrm{H}\right.$, cis- $\mathrm{C}^{7} \mathrm{H},{ }^{2} J=16.1,{ }^{3} \mathrm{~J}=$ $5.0 \mathrm{~Hz}), 2.99$ d. d $\left(1 \mathrm{H}\right.$, trans $-\mathrm{C}^{7} \mathrm{H},{ }^{2} J=16.1,{ }^{3} J=7.1$ $\mathrm{Hz}), 3.68 \mathrm{~s}(3 \mathrm{H}, \mathrm{MeO}), 3.79 \mathrm{~d} . \mathrm{d}\left(1 \mathrm{H}, \mathrm{C}^{8} \mathrm{H},{ }^{3} \mathrm{~J}=7.1,{ }^{3} \mathrm{~J}=\right.$ $5.0 \mathrm{~Hz}), 3.96 \mathrm{q}\left(2 \mathrm{H}, \mathrm{OC}_{2} \mathrm{CH}_{3},{ }^{3} \mathrm{~J}=7.0 \mathrm{~Hz}\right), 5.29-5.44 \mathrm{~m}$ $\left(4 \mathrm{H}, \mathrm{NCH}_{2} \mathrm{NCH}_{2} \mathrm{~S}\right), 6.43 \mathrm{~d}$. d $\left(1 \mathrm{H}, \mathrm{H}-\mathrm{Ar},{ }^{3} J=8.1,{ }^{4} J=\right.$ $2.0 \mathrm{~Hz}), 6.76-6.79 \mathrm{~m}(2 \mathrm{H}, \mathrm{H}-\mathrm{Ar}), 6.91 \mathrm{~d}\left(1 \mathrm{H}, \mathrm{H}-\mathrm{Ar},{ }^{4} J=\right.$ $2.5 \mathrm{~Hz}), 7.06 \mathrm{~d}\left(1 \mathrm{H}, \mathrm{H}-\mathrm{Ar},{ }^{3} \mathrm{~J}=8.3 \mathrm{~Hz}\right) .{ }^{13} \mathrm{C}$ DEPTQ NMR spectrum, $\delta_{\mathrm{C}}$, ppm: $14.8 *\left(\mathrm{CH}_{3} \mathrm{CH}_{2} \mathrm{O}\right), 18.4 *\left(\mathrm{CH}_{3} \mathrm{Ar}\right)$, 
19.9* $\left(\mathrm{CH}_{3} \mathrm{Ar}\right), 37.4^{*}\left(\mathrm{C}^{8} \mathrm{H}\right), 38.1\left(\mathrm{C}^{7} \mathrm{H}_{2}\right), 52.6\left(\mathrm{C}^{2} \mathrm{H}_{2}\right)$, 55.4* $\left(\mathrm{CH}_{3} \mathrm{O}\right), 57.9\left(\mathrm{C}^{4} \mathrm{H}_{2}\right), 63.6\left(\mathrm{CH}_{3} \mathrm{CH}_{2} \mathrm{O}\right), 89.1\left(\mathrm{C}^{9}\right)$, 111.1* (CH Ar), 112.7* (CH Ar), 113.9* (CH Ar), 117.6 $(\mathrm{C} \equiv \mathrm{N}), 117.8^{*}(\mathrm{CH} \mathrm{Ar}), 118.3^{*}(\mathrm{CH} \mathrm{Ar}), 129.2(\mathrm{C} \mathrm{Ar})$, 130.4* (CH Ar), 132.0 (C Ar), 137.2 (C Ar), 141.6 (C Ar), 147.3 (C $\mathrm{C}^{4}$-OEt C-Ar), 148.9 (C ${ }^{3}$-OMe C-Ar), 149.1 $\left(\mathrm{C}^{9 \mathrm{a}}\right), 167.3(\mathrm{C}=\mathrm{O})$. Mass spectrum (HRMS ESI-TOF), $m / z$ : $472.1656[M+\mathrm{Na}]^{+}$(calcd. for $\mathrm{C}_{25} \mathrm{H}_{27} \mathrm{~N}_{3} \mathrm{NaO}_{3} \mathrm{~S}$ : 472.1665), $540.1520[M+\mathrm{HCOONa}+\mathrm{Na}]^{+}$(calcd. for $\left.\mathrm{C}_{26} \mathrm{H}_{28} \mathrm{~N}_{3} \mathrm{Na}_{2} \mathrm{O}_{5} \mathrm{~S}: 540.1545\right)$.

3-(2,4-Dimethoxyphenyl)-8-(3-methoxy-4-ethoxyphenyl)-6-oxo-2,3,4,6,7,8-hexahydropyrido[2,1- $b]$ $[\mathbf{1}, 3,5]$ thiadiazine-9-carbonitrile (8c). Yield 91\%, beige crystals. IR spectrum, $v, \mathrm{~cm}^{-1}: 2201 \mathrm{~s}(\mathrm{C} \equiv \mathrm{N}), 1684 \mathrm{~s}$ $(\mathrm{C}=\mathrm{O}) .{ }^{1} \mathrm{H}$ NMR spectrum, $\delta$, ppm: $1.30 \mathrm{t}\left(3 \mathrm{H}, \mathrm{OCH}_{2} \mathrm{CH}_{3}\right.$, $\left.{ }^{3} J=7.0 \mathrm{~Hz}\right), 2.69 \mathrm{~d} . \mathrm{d}\left(1 \mathrm{H}\right.$, cis $-\mathrm{C}^{7} \mathrm{H},{ }^{2} J=16.1,{ }^{3} \mathrm{~J}=$ $4.8 \mathrm{~Hz}), 3.02 \mathrm{~d}$. d $\left(1 \mathrm{H}\right.$, trans $\left.-\mathrm{C}^{7} \mathrm{H},{ }^{2} J=16.1,{ }^{3} J=7.1 \mathrm{~Hz}\right)$, $3.71 \mathrm{~s}(3 \mathrm{H}, \mathrm{MeO}), 3.74 \mathrm{~s}(3 \mathrm{H}, \mathrm{MeO}), 3.79 \mathrm{~s}(3 \mathrm{H}, \mathrm{MeO})$, $3.83-3.85 \mathrm{~m}\left(1 \mathrm{H}, \mathrm{C}^{8} \mathrm{H}\right), 3.96 \mathrm{q}\left(2 \mathrm{H}, \mathrm{OC}_{2} \mathrm{CH}_{3},{ }^{3} \mathrm{~J}=\right.$ $7.0 \mathrm{~Hz}), 5.10-5.28 \mathrm{~m}\left(4 \mathrm{H}, \mathrm{NCH}_{2} \mathrm{NCH}_{2} \mathrm{~S}\right), 6.45-6.48 \mathrm{~m}$ $\left(2 \mathrm{H}, \mathrm{H}^{4} \mathrm{~N}-\mathrm{Ar}+\mathrm{H}^{6} \mathrm{C}-\mathrm{Ar}\right), 6.60 \mathrm{~d}\left(1 \mathrm{H}, \mathrm{H}^{3} \mathrm{~N}-\mathrm{Ar},{ }^{4} \mathrm{~J}=\right.$ $2.3 \mathrm{~Hz}), 6.75 \mathrm{~d}\left(1 \mathrm{H}, \mathrm{H}^{5} \mathrm{C}-\mathrm{Ar},{ }^{3} J=8.3 \mathrm{~Hz}\right), 6.79 \mathrm{~d}(1 \mathrm{H}$, $\left.\mathrm{H}^{2} \mathrm{C}-\mathrm{Ar},{ }^{4} J=1.4 \mathrm{~Hz}\right), 7.01 \mathrm{~d}\left(1 \mathrm{H}, \mathrm{H}^{6} \mathrm{~N}-\mathrm{Ar},{ }^{3} J=8.7 \mathrm{~Hz}\right)$. ${ }^{13} \mathrm{C}$ DEPTQ NMR spectrum, $\delta_{\mathrm{C}}$, ppm: $14.8^{*}\left(\mathrm{CH}_{3} \mathrm{CH}_{2} \mathrm{O}\right)$, 37.3* $\left(\mathrm{C}^{8} \mathrm{H}\right), 38.2\left(\mathrm{C}^{7} \mathrm{H}_{2}\right), 54.7\left(\mathrm{C}^{2} \mathrm{H}_{2}\right), 55.3 *\left(\mathrm{CH}_{3} \mathrm{O}\right.$ C-Ar), 55.4* (4- $\mathrm{CH}_{3} \mathrm{O}$ N-Ar), 55.7* (2- $\left.\mathrm{CH}_{3} \mathrm{O} \mathrm{N}-\mathrm{Ar}\right)$, $60.4\left(\mathrm{C}^{4} \mathrm{H}_{2}\right), 63.6\left(\mathrm{CH}_{3} \underline{\mathrm{CH}}_{2} \mathrm{O}\right), 88.7\left(\mathrm{C}^{9}\right), 100.0^{*}\left(\mathrm{C}^{3} \mathrm{H}\right.$ $\mathrm{N}-\mathrm{Ar}), 104.2 *\left(\mathrm{C}^{5} \mathrm{HN}-\mathrm{Ar}\right), 111.0^{*}\left(\mathrm{C}^{2} \mathrm{H} \mathrm{C}-\mathrm{Ar}\right), 112.6 *$ $\left(\mathrm{C}^{5} \mathrm{H} \mathrm{C}-\mathrm{Ar}\right), 117.9(\mathrm{C} \equiv \mathrm{N}), 118.2 *\left(\mathrm{C}^{6} \mathrm{H} \mathrm{C}-\mathrm{Ar}\right), 121.0 *$ (C ${ }^{6} \mathrm{H}$ N-Ar), 127.3 (C $\left.{ }^{1} \mathrm{~N}-\mathrm{Ar}\right), 132.1$ (C $\left.\mathrm{C}^{1} \mathrm{C}-\mathrm{Ar}\right), 147.3$ (C $\mathrm{C}^{4}$-OEt C-Ar), 149.0 (C $\mathrm{C}^{3}$-OMe C-Ar), $149.3\left(\mathrm{C}^{9 \mathrm{a}}\right), 152.8$ $\left(\mathrm{C}^{2}-\mathrm{OMe} \mathrm{N}-\mathrm{Ar}\right), 157.1$ (C4-OMe N-Ar), $167.3(\mathrm{C}=\mathrm{O})$. Mass spectrum (HRMS ESI-TOF), $m / z: 504.1552[M+$ $\mathrm{Na}]^{+}$(calcd. for $\mathrm{C}_{25} \mathrm{H}_{27} \mathrm{~N}_{3} \mathrm{NaO}_{5} \mathrm{~S}$ : 504.1564), 572.1417 $[M+\mathrm{HCOONa}+\mathrm{Na}]^{+}$(calcd. for $\mathrm{C}_{26} \mathrm{H}_{28} \mathrm{~N}_{3} \mathrm{Na}_{2} \mathrm{O}_{7} \mathrm{~S}$ : 572.1444).

2-\{[4-(4-Hydroxy-3-methoxyphenyl)-5-oxo-3cyano-1,4,5,6,7,8-hexahydroquinolin-2-yl]thio $\}$ $\mathrm{N}$-(4-fluorophenyl)acetamide (9). A mixture of cyanothioacetamide $\mathbf{1}(2.0 \mathrm{~g}, 20 \mathrm{mmol})$, vanillin (3.04 g, $20 \mathrm{mmol}$ ), $25 \mathrm{~mL}$ of ethanol and 3 drops of piperidine was stirred until the starting reagents consumed and an orange Knoevenagel condensation product formed. To the resulting suspension were added 1,3-cyclohexanedione $(2.24 \mathrm{~g}, 20 \mathrm{mmol})$ and piperidine $(2.5 \mathrm{~mL}, 25 \mathrm{mmol})$. The mixture was stirred for $8 \mathrm{~h}$, then acidified with $10 \% \mathrm{HCl}$ in ethanol to $\mathrm{pH} 5$. After $12 \mathrm{~h}$, the precipitate was filtered off and washed with cooled alcohol. 4-(4-Hydroxy-3- methoxyphenyl)-2-mercapto-5-oxo-1,4,5,6,7,8-hexahydroquinoline-3-carbonitrile $\mathbf{1 6}$ was obtained (yield $67 \%$ ), which was used in the next step without additional purification.

To a suspension of 2-mercaptoquinoline 16 (660 mg, $2 \mathrm{mmol}$ ) in $15 \mathrm{~mL}$ of $85 \%$ ethanol were added an aqueous $10 \% \mathrm{KOH}$ solution $(1.05 \mathrm{~mL}, 2 \mathrm{mmol})$ and $\mathrm{N}$-(4-fluorophenyl)- $\alpha$-chloroacetamide (375 mg, $2 \mathrm{mmol}$ ). The resulting mixture was stirred for $12 \mathrm{~h}$ at $25^{\circ} \mathrm{C}$. The precipitated compound 9 was filtered off and recrystallized from an acetone-ethanol mixture. Yield $86 \%$, beige powder. IR spectrum, $v, \mathrm{~cm}^{-1}: 3433 \mathrm{~s}, 3309 \mathrm{~s}$, 3282 br, 3221 br, $3095(\mathrm{O}-\mathrm{H}, \mathrm{N}-\mathrm{H}), 2189$ s $(\mathrm{C} \equiv \mathrm{N})$, $1672 \mathrm{~s}(\mathrm{C}=\mathrm{O}), 1651 \mathrm{~s}[\mathrm{C}(\mathrm{O}) \mathrm{NH}] .{ }^{1} \mathrm{H}$ NMR spectrum, $\delta$, ppm: $1.75-1.93 \mathrm{~m}\left(2 \mathrm{H}, \mathrm{C}^{7} \mathrm{H}_{2}\right), 2.21-2.25 \mathrm{~m}\left(2 \mathrm{H}, \mathrm{C}^{6} \mathrm{H}_{2}\right)$, 2.49-2.59 m (2H, $\left.\mathrm{C}^{8} \mathrm{H}_{2}\right), 3.70 \mathrm{~s}(3 \mathrm{H}, \mathrm{MeO}), 3.90 \mathrm{q}(2 \mathrm{H}$, $\left.\mathrm{SCH}_{2},{ }^{2} J=14.7 \mathrm{~Hz}\right), 4.40 \mathrm{~s}\left(1 \mathrm{H}, \mathrm{H}^{4}\right), 6.53 \mathrm{~d} . \mathrm{d}(1 \mathrm{H}$, $\left.\mathrm{H}^{6} \mathrm{Ar},{ }^{3} J=8.1,{ }^{4} J=1.7 \mathrm{~Hz}\right), 6.64 \mathrm{~d}\left(1 \mathrm{H}, \mathrm{H}^{5} \mathrm{Ar},{ }^{3} J=\right.$ $8.1 \mathrm{~Hz}), 6.70 \mathrm{~d}\left(1 \mathrm{H}, \mathrm{H}^{2} \mathrm{Ar},{ }^{4} J=1.7 \mathrm{~Hz}\right), 7.14-7.18 \mathrm{~m}(2 \mathrm{H}$, $\left.\mathrm{H}^{3}, \mathrm{H}^{5} 4-\mathrm{FC}_{6} \mathrm{H}_{4}\right), 7.52-7.56 \mathrm{~m}\left(2 \mathrm{H}, \mathrm{H}^{2}, \mathrm{H}^{6} 4-\mathrm{FC}_{6} \mathrm{H}_{4}\right)$, $8.86 \mathrm{~s}(1 \mathrm{H}, \mathrm{OH}), 9.99 \mathrm{~s}\left(1 \mathrm{H}, \mathrm{N}^{1} \mathrm{H}\right), 10.39 \mathrm{~s}[1 \mathrm{H}, \mathrm{C}(\mathrm{O})$ $\mathrm{NH}] .{ }^{13} \mathrm{C}$ DEPTQ NMR spectrum, $\delta_{\mathrm{C}}$, ppm: $20.7\left(\mathrm{C}^{7} \mathrm{H}_{2}\right)$, $26.3\left(\mathrm{C}^{8} \mathrm{H}_{2}\right), 36.7\left(\mathrm{C}^{6} \mathrm{H}_{2}\right), 36.8\left(\mathrm{SCH}_{2}\right), 38.6^{*}\left(\mathrm{C}^{4} \mathrm{H}\right)$, 55.5* (OMe), $92.0\left(\mathrm{C}^{3}\right), 109.2\left(\mathrm{C}^{4 \mathrm{a}}\right), 111.5^{*}\left(\mathrm{C}^{2} \mathrm{H} \mathrm{Ar}\right)$, $115.4 *\left(\mathrm{C}^{5} \mathrm{H} \mathrm{Ar}\right), 115.5^{*} \mathrm{~d}\left(\mathrm{C}^{3} \mathrm{H}, \mathrm{C}^{5} \mathrm{H} 4-\mathrm{FC}_{6} \mathrm{H}_{4},{ }^{2} J_{\mathrm{CF}}=\right.$ $22.4 \mathrm{~Hz}), 119.1(\mathrm{C} \equiv \mathrm{N}), 119.4 *\left(\mathrm{C}^{6} \mathrm{H} \mathrm{Ar}\right), 121.4 * \mathrm{~d}\left(\mathrm{C}^{2} \mathrm{H}\right.$, $\left.\mathrm{C}^{6} \mathrm{H} 4-\mathrm{FC}_{6} \mathrm{H}_{4},{ }^{3} J_{\mathrm{CF}}=8.1 \mathrm{~Hz}\right), 134.8 \mathrm{~d}\left(\mathrm{C}^{1} 4-\mathrm{FC}_{6} \mathrm{H}_{4}\right.$, $\left.{ }^{4} J_{\mathrm{CF}}=2.4 \mathrm{~Hz}\right), 136.1\left(\mathrm{C}^{1} \mathrm{Ar}\right), 142.1\left(\mathrm{C}^{2}\right), 145.5$ ( $\mathrm{C}^{4}-\mathrm{OH}$ Ar), 147.4 ( $\left.\mathrm{C}^{5}-\mathrm{OMe} \mathrm{Ar}\right), 151.3\left(\mathrm{C}^{8 \mathrm{a}}\right), 158.3$ d $\left(\mathrm{C}^{4} 4-\mathrm{FC}_{6} \mathrm{H}_{4},{ }^{1} J_{\mathrm{CF}}=240.6 \mathrm{~Hz}\right), 166.6[\mathrm{C}(\mathrm{O}) \mathrm{NH}]$, $194.9(\mathrm{C}=\mathrm{O})$. Mass spectrum (HRMS ESI-TOF), $m / z$ : $502.1201[M+\mathrm{Na}]^{+}$(calcd. for $\mathrm{C}_{25} \mathrm{H}_{22} \mathrm{FN}_{3} \mathrm{NaO}_{4} \mathrm{~S}$ : 502.1207).

Ethyl 2-ox0-4-(2-fluorophenyl)-5-cyano-6-[(2\{(2-ethylphenyl)amino\}-2-oxoethyl)thio]-1,2,3,4tetrahydropyridine-3-carboxylic acid (10). A mixture of cyanothioacetamide $1(2.0 \mathrm{~g}, 20 \mathrm{mmol})$, 2 -fluorobenzaldehyde $(2.1 \mathrm{~mL}, 20 \mathrm{mmol})$, and diethylmalonate $(3.05 \mathrm{ml}, 20 \mathrm{mmol})$ was stirred in $25 \mathrm{~mL}$ of ethanol in the presence of an excess of $\mathrm{N}$-methylmorpholine $(25 \mathrm{mmol})$ for 7 days. The precipitate of tetrahydropyridine-2-thiolate 17 was filtered off, washed with acetone, and dried in air. Yield $44 \%$, white powder. Thiolate 17 was used without further purification.

A mixture of thiolate $17(2 \mathrm{mmol})$ and $\alpha$-chloro$\mathrm{N}$-(2-ethylphenyl)acetamide $(2 \mathrm{mmol})$ was heated in $15 \mathrm{~mL}$ of $85 \%$ ethanol until the starting reagents were 
completely dissolved and a clear solution was formed. After $24 \mathrm{~h}$, the crystallized product $\mathbf{1 0}$ was filtered off, washed with ethanol and petroleum ether. Yield 83\%, beige powder. IR spectrum, $v, \mathrm{~cm}^{-1}: 3325 \mathrm{~s}(\mathrm{~N}-\mathrm{H}), 2208 \mathrm{~s}$ $(\mathrm{C} \equiv \mathrm{N}), 1732 \mathrm{~s}(\mathrm{C}=\mathrm{O}), 1693 \mathrm{~s}[\mathrm{C}(\mathrm{O}) \mathrm{NH}], 1655 \mathrm{~s}[\mathrm{C}(\mathrm{O})$ $\mathrm{NH}] .{ }^{1} \mathrm{H}$ NMR spectrum, $\delta$, ppm: $0.97 \mathrm{t}\left(3 \mathrm{H}, \mathrm{ArCH}_{2} \mathrm{CH}_{3}\right.$, $\left.{ }^{3} J=7.8 \mathrm{~Hz}\right), 1.12 \mathrm{t}\left(3 \mathrm{H}, \mathrm{OCH}_{2} \mathrm{CH}_{3},{ }^{3} J=7.1 \mathrm{~Hz}\right), 2.59 \mathrm{q}$ $\left(2 \mathrm{H}, \mathrm{ArCH}_{2} \mathrm{CH}_{3}, 3 \mathrm{~J}=7.8 \mathrm{~Hz}\right), 3.97-4.04 \mathrm{~m}\left(5 \mathrm{H}, \mathrm{SCH}_{2}\right.$, $\left.\mathrm{H}^{4}, \mathrm{OCH}_{2}\right), 4.55 \mathrm{~d}\left(1 \mathrm{H}, \mathrm{H}^{3},{ }^{3} \mathrm{~J}=10.8 \mathrm{~Hz}\right), 7.18-7.22 \mathrm{~m}$ (3H, H-Ar), 7.24-7.27 m (2H, H-Ar), 7.31-7.42 m (3H, $\mathrm{H}-\mathrm{Ar}), 9.79 \mathrm{~s}(1 \mathrm{H}, \mathrm{NHAr}), 11.28 \mathrm{~s}(1 \mathrm{H}, \mathrm{NH}) .{ }^{13} \mathrm{C}$ DEPTQ NMR spectrum, $\delta_{\mathrm{C}}, \mathrm{ppm}: 13.7^{*}\left(\mathrm{C}_{3} \mathrm{CH}_{2} \mathrm{Ar}\right), 14.83^{*}$ $\left(\underline{\mathrm{CH}}_{3} \mathrm{CH}_{2} \mathrm{O}\right), 23.6\left(\mathrm{CH}_{3} \underline{\mathrm{CH}}_{2} \mathrm{Ar}\right), 35.3\left(\mathrm{SCH}_{2}\right), 36.8^{*} \mathrm{~d}$ $\left(\mathrm{C}^{4} \mathrm{H},{ }^{3} J_{\mathrm{CF}}=1.7 \mathrm{~Hz}\right), 52.1 *\left(\mathrm{C}^{3}\right), 61.2\left(\mathrm{CH}_{3} \mathrm{CH}_{2} \mathrm{O}\right), 91.2$ $\left(\mathrm{C}^{5}\right), 115.9^{*} \mathrm{~d}\left(\mathrm{C}^{3} \mathrm{H} 2-\mathrm{FC}_{6} \mathrm{H}_{4},{ }^{2} J_{\mathrm{CF}}=21.5 \mathrm{~Hz}\right), 116.7$ $(\mathrm{C} \equiv \mathrm{N}), 123.7 \mathrm{~d}\left(\mathrm{C}^{1} 2-\mathrm{FC}_{6} \mathrm{H}_{4},{ }^{2} J_{\mathrm{CF}}=13.4 \mathrm{~Hz}\right), 124.9^{*} \mathrm{~d}$ $\left(\mathrm{C}^{5} \mathrm{H} 2-\mathrm{FC}_{6} \mathrm{H}_{4},{ }^{4} J_{\mathrm{CF}}=3.1 \mathrm{~Hz}\right), 126.01 *(\mathrm{CH} \mathrm{N}-\mathrm{Ar})$, 126.04* (CH N-Ar), 126.3* (CH N-Ar), 128.6* (CH $\mathrm{N}-\mathrm{Ar}), 129.6^{*} \mathrm{~d}\left(\mathrm{C}^{6} \mathrm{H} 2-\mathrm{FC}_{6} \mathrm{H}_{4},{ }^{3} J_{\mathrm{CF}}=3.3 \mathrm{~Hz}\right), 130.5^{*} \mathrm{~d}$ $\left(\mathrm{C}^{4} \mathrm{H} 2-\mathrm{FC}_{6} \mathrm{H}_{4},{ }^{3} J_{\mathrm{CF}}=8.4 \mathrm{~Hz}\right), 134.8\left(\mathrm{C}^{2} \mathrm{~N}-\mathrm{Ar}\right), 138.2$ $\left(\mathrm{C}^{1} \mathrm{~N}-\mathrm{Ar}\right), 147.2\left(\mathrm{C}^{6}\right), 160.2 \mathrm{~d}\left(\mathrm{C}^{2} 2-\mathrm{FC}_{6} \mathrm{H}_{4},{ }^{1} J_{\mathrm{CF}}=\right.$ $246.3 \mathrm{~Hz}), 165.2[\mathrm{C}(\mathrm{O}) \mathrm{NH}], 166.8[\mathrm{C}(\mathrm{O}) \mathrm{NH}], 167.3$ $\left(\mathrm{CO}_{2} \mathrm{Et}\right)$. Mass spectrum (HRMS ESI-TOF), $\mathrm{m} / \mathrm{z}$ : $504.1354[M+\mathrm{Na}]^{+}$(calcd. for $\mathrm{C}_{25} \mathrm{H}_{24} \mathrm{FN}_{3} \mathrm{NaO}_{4} \mathrm{~S}$ : 504.1364).

7,7-Dimethyl-2-methylthio-4-(3,4-dimethoxyphenyl)-5-oxo-1,4,5,6,7,8-hexahydroquinoline-3carbonitrile (11). A mixture of cyanothioacetamide 1 (200 mg, $2 \mathrm{mmol}$ ), veratraldehyde (333 mg, $2 \mathrm{mmol}$ ), and piperidine ( 1 drop) in $5 \mathrm{~mL}$ of ethanol was stirred for 5 min until an orange precipitate of the Knoevenagel condensation product was formed. To the resulting suspension, dimedone (5,5-dimethylcyclohexane-1,3dione) $(280 \mathrm{mg}, 2 \mathrm{mmol})$ and piperidine $(0.25 \mathrm{~mL}$, $2.5 \mathrm{mmol})$ were added with vigorous stirring. The resulting mixture was stirred until dissolved, filtered through a paper filter and kept for $24 \mathrm{~h}$; in this case, the formation of a white fine-crystalline precipitate of the Michael adduct can be observed. Iodomethane $(0.15 \mathrm{~mL}$, $2.4 \mathrm{mmol}$ ) and $4 \mathrm{~mL}$ of $70 \%$ ethanol were added to the reaction mixture. The mixture was slowly heated to boiling with stirring, boiled for $10 \mathrm{~min}$, then filtered through a paper filter and allowed to crystallize. The precipitate of quinoline $\mathbf{1 1}$ was filtered off, washed with ethanol, petroleum ether, and dried in air. Yield 74\%, beige powder. IR spectrum, $\mathrm{v}, \mathrm{cm}^{-1}: 3252,3173 \mathrm{w}(\mathrm{N}-\mathrm{H})$, $2195 \mathrm{~s}(\mathrm{C} \equiv \mathrm{N}), 1606$ br. $\mathrm{s}(\mathrm{C}=\mathrm{O}, \mathrm{C}=\mathrm{C}) .{ }^{1} \mathrm{H}$ NMR spectrum, $\delta$, ppm: $0.91 \mathrm{~s}\left(3 \mathrm{H}, \mathrm{C}^{7} \mathrm{CH}_{3}\right), 1.02 \mathrm{~s}\left(3 \mathrm{H}, \mathrm{C}^{7} \mathrm{CH}_{3}\right), 2.02$ d $\left(1 \mathrm{H}, \mathrm{C}^{6} \mathrm{H}_{2},{ }^{2} J=16.4 \mathrm{~Hz}\right.$, AB-system $), 2.21 \mathrm{~d}(1 \mathrm{H}$, $\mathrm{C}^{6} \mathrm{H}_{2},{ }^{2} \mathrm{~J}=16.4 \mathrm{~Hz}, \mathrm{AB}$-system $), 2.43 \mathrm{q}\left(2 \mathrm{H}, \mathrm{C}^{8} \mathrm{H}_{2},{ }^{2} \mathrm{~J}=\right.$ $17.6 \mathrm{~Hz}), 2.48 \mathrm{~s}\left(3 \mathrm{H}, \mathrm{SCH}_{3}\right), 3.67 \mathrm{~s}\left(3 \mathrm{H}, \mathrm{OCH}_{3}\right), 3.69 \mathrm{~s}$ $\left(3 \mathrm{H}, \mathrm{OCH}_{3}\right), 4.38 \mathrm{~s}\left(1 \mathrm{H}, \mathrm{H}^{4}\right), 6.63 \mathrm{~d} . \mathrm{d}\left(1 \mathrm{H}, \mathrm{H}^{6} \mathrm{Ar},{ }^{3} J=\right.$ $\left.8.3,{ }^{4} J=2.0 \mathrm{~Hz}\right), 6.69 \mathrm{~d}\left(1 \mathrm{H}, \mathrm{H}^{2} \mathrm{Ar},{ }^{4} J=2.0 \mathrm{~Hz}\right), 6.86 \mathrm{~d}$ $\left(1 \mathrm{H}, \mathrm{H}^{5} \mathrm{Ar},{ }^{3} J=8.3 \mathrm{~Hz}\right), 9.61 \mathrm{~s}(1 \mathrm{H}, \mathrm{NH}) .{ }^{13} \mathrm{C}$ DEPTQ NMR spectrum, $\delta_{\mathrm{C}}, \mathrm{ppm}: 15.8^{*}\left(\mathrm{SCH}_{3}\right), 26.3^{*}\left(\mathrm{C}^{7} \mathrm{CH}_{3}\right)$, $29.1^{*}\left(\mathrm{C}^{7} \mathrm{CH}_{3}\right), 32.0\left(\mathrm{C}^{7}\right), 38.6^{*}\left(\mathrm{C}^{4} \mathrm{H}\right), 39.3\left(\mathrm{C}^{8} \mathrm{H}_{2}\right), 50.1$ $\left(\mathrm{C}^{6} \mathrm{H}_{2}\right), 55.4^{*}(\mathrm{OMe}), 55.5^{*}(\mathrm{OMe}), 90.3\left(\mathrm{C}^{3}\right), 108.2$ $\left(\mathrm{C}^{4 \mathrm{a}}\right), 110.8^{*}$ ( $\left.\mathrm{C}^{2} \mathrm{H} \mathrm{C}-\mathrm{Ar}\right), 111.8^{*}\left(\mathrm{C}^{5} \mathrm{H} \mathrm{C}-\mathrm{Ar}\right), 118.95^{*}$ (C ${ }^{6} \mathrm{H}$ C-Ar $), 118.99(\mathrm{C} \equiv \mathrm{N}), 137.7\left(\mathrm{C}^{1} \mathrm{C}-\mathrm{Ar}\right), 144.5\left(\mathrm{C}^{8 \mathrm{a}}\right)$, 147.7 (COMe), 148.6 (COMe), 149.6 ( $\left.\mathrm{C}^{2}\right), 194.6(\mathrm{C}=\mathrm{O})$. Mass spectrum (HRMS ESI-TOF), $m / z: 407.1393[M+$ $\mathrm{Na}]^{+}$(calcd. for $\mathrm{C}_{21} \mathrm{H}_{24} \mathrm{~N}_{2} \mathrm{NaO}_{3} \mathrm{~S}$ : 407.1400).

The study of analgesic activity was carried out on 56 white outbred sexually mature rats of both sexes weighing $160-200 \mathrm{~g}$ in the autumn-winter period. Throughout the experiment, the animals were kept in a vivarium, the diet was standard, there were no more than 6 individuals in the cage, which corresponds to the guidelines for ethical review of biomedical research $[68,69]$. In the pre-experimental period, a thorough examination of the animals was carried out, special attention was paid to their weight, age, physical activity and the animal coat. Based on these data, laboratory rats were evenly divided into groups: intact, control (with simulated orofacial trigeminal pain and heat tail immersion), reference (ketorolac), and 6 experimental groups according to the number of $\alpha$-cyanothioacetamide derivatives studied.

Proceeding from the principles of humanity, the experiment was carried out with the minimum number of animals ( 6 per group) permissible for statistical processing and obtaining reliable results, as well as the minimum number of experimental groups to achieve the set goals and objectives. The assessment of analgesic activity was carried out in the test of orofacial trigeminal pain, which was simulated by the subcutaneous injection of $0.1 \mathrm{~mL}$ of a $5 \%$ formalin solution into the vibrissa area in laboratory rats [69]. Within $20 \mathrm{~min}$ (for 10, 15 and $20 \mathrm{~min}$ ) after the introduction of algogen, the number of scratching movements with the front paws of the orofacial area was counted. Ketorolac at a dose of $0.1 \mathrm{mg} / \mathrm{kg}$ was used as a reference drug for the reference group of rats; 6 samples of the tested pyridine derivatives were injected intragastrically at a dose of $5 \mathrm{mg} / \mathrm{kg} 1.5$ hours before the administration of the algogen (formalin) used.

To evaluate thermal pain, we chose the method of thermal tail immersion, based on the spinal flexor reflex 
in response to immersion of the tail in hot water. Painful irritation was simulated by immersing the tail in a vessel with water heated to $50-54^{\circ} \mathrm{C}$, while measuring the value of the latent period of the reaction. Analgesic activity was assessed by lengthening the tail flick reaction time.

The results were statistically processed using the Statistica 10.0 software package. Since the study was carried out on the smallest possible number of laboratory animals, nonparametric methods were used in the analysis of the numerical results of the experimental part of the work. In order to determine the reliability of differences in statistical data processing, a nonparametric Wilcoxon matched-pairs signed ranks test was used with preliminary determination of valid intervals of values for each of the studied indicators. In this case, the samples were assessed as continuous, sufficient in terms of the variability of the feature [70]. The significance of differences between the values of the control and experimental groups was calculated on the basis of the data obtained (median, quartile, mean values, standard deviation).

This work was performed in compliance with all applicable international, national and institutional guidelines for the care and use of animals.

\section{FUNDING}

This work was financially supported by the Kuban Science Foundation within the framework of the scientific project MFI-20.1-26/20 (application no. MFI-20.1/45), as well as the Ministry of Education and Science of the Russian Federation (topic 0795-2020-0031).

\section{CONFLICT OF INTEREST}

The authors declare no conflicts of interest.

\section{SUPPLEMENTARY INFORMATION}

The online version contains supplementary material available at https://doi.org/10.1134/S107036322102002X.

\section{REFERENCES}

1. Abdel-Galil, F.M., Sherif, S.M., and Elnagdi, M.H., Heterocycles, 1986, vol. 24, no. 7, p. 2023. https://doi.org/10.3987/R-1986-07-2023

2. Litvinov, V.P., Russ. Chem.Rev., 1999, vol.68, no.9, p. 737. https://doi.org/10.1070/RC1999v068n09ABEH000533

3. Dyachenko, V.D., Dyachenko, I.V., and Nenajdenko, V.G., Russ. Chem. Rev., 2018, vol. 87, no. 1, p. 1. https://doi.org/10.1070/RCR4760

4. Magerramov, A.M., Shikhaliev, N.G., Dyachenko, V.D., Dyachenko, I.V., and Nenajdenko, V.G., $\alpha$-Tsyanotioatsetamid ( $\alpha$-Cyanothioacetamide), Moscow: Tekhnosfera, 2018.

5. Litvinov, V.P., Rodinovskaya, L.A., Sharanin, Yu.A.,
Shestopalov, A.M., and Senning, A., J. Sulfur Chem., 1992, vol. 13, no. 1 , p. 1.

https://doi.org/10.1080/01961779208048951

6. Litvinov, V.P., Phosphorus, Sulfur, Silicon Relat. Elem., 1993, vol. 74, no. 1, p. 139.

https://doi.org/10.1080/10426509308038105

7. Litvinov, V.P., Russ. Chem. Bull., 1998, vol. 47, no. 11, p. 2053.

https://doi.org/10.1007/BF02494257

8. Litvinov, V.P., Russ. Chem. Rev., 2006, vol. 75, no. 7, p. 577. https://doi.org/10.1070/RC2006v075n07ABEH003619

9. Litvinov, V.P., Promonenkov, V.K., Sharanin, Yu.A., and Shestopalov, A.M., Itogi Nauki i Tekhniki. Ser. Org. Khim., Moscow: VINITI, 1989, vol. 17, p. 72.

10. El-Sayed, H.A., Moustafa, A.H., Said, S.A., Assy, M.G., and Amr, A.E.-G.E., Synth. Commun., 2018, vol. 48, no. 20 , p. 2615.

https://doi.org/10.1080/00397911.2018.1496262

11. Salem, M.A., Helel, M.H., Gouda, M.A., Ammar, Y.A., and El-Gaby, M.S.A., Synth. Commun., 2018, vol. 48, no. 4 , p. 345.

https://doi.org/10.1080/00397911.2017.1394468

12. Dotsenko, V.V., Buryi, D.S., Lukina, D.Yu., and Krivokolysko, S.G., Russ. Chem. Bull., 2020, vol. 69, no. 10 , p. 1829.

https://doi.org/10.1007/s11172-020-2969-2

13. Dotsenko, V.V., Krivokolysko, S.G., Krivokolysko, B.S., and Frolov, K.A., Russ. J. Gen. Chem., 2018, vol. 88, no. 4, p. 682.

https://doi.org/10.1134/S1070363218040114

14. Buryi, D.S., Dotsenko, V.V., Aksenov, N.A., and Aksenova, I.V., Russ. J. Gen. Chem., 2019, vol. 89, no. 9, p. 1744. https://doi.org/10.1134/S1070363219090032

15. Buryi, D.S., Dotsenko, V.V., Aksenov, N.A., Aksenova, I.V., Krivokolysko, S.G., and Dyadyuchenko, L.V., Russ. J. Gen. Chem., 2019, vol. 89, no. 8, p. 1575. https://doi.org/10.1134/S1070363219080061

16. Chigorina, E.A., Bespalov, A.V., and Dotsenko, V.V., Russ. J. Gen. Chem., 2019, vol. 89, no. 10, p. 2018. https://doi.org/10.1134/S1070363219100062

17. Buryi, D.S., Dotsenko, V.V., Levashov, A.S., Lukina, D.Yu., Strelkov, V.D., Aksenov, N.A., Aksenova, I.V., and Netreba, E.E., Russ. J. Gen. Chem., 2019, vol. 89 , no. 5 , p. 886 .

https://doi.org/10.1134/S1070363219050050

18. Dotsenko, V.V., Buryi, D.S., Lukina, D.Yu., Stolyarova, A.N., Aksenov, N.A., Aksenova, I.V., Strelkov, V.D., and Dyadyuchenko, L.V., Monatsh. Chem., 2019, vol. 150 , no. 11, p. 1973.

https://doi.org/10.1007/s00706-019-02505-4

19. Stroganova, T.A., Vasilin, V.K., Dotsenko, V.V., Aksenov, N.A., and Krapivin, G.D., Tetrahedron 
Lett., 2019, vol. 60, p. 997.

https://doi.org/10.1016/j.tetlet.2019.03.012

20. Dotsenko, V.V., Muraviev, V.S., Lukina, D.Yu., Strelkov, V.D., Aksenov, N.A., Aksenova, I.V., Krapivin, G.D., and Dyadyuchenko, L.V., Russ. J. Gen. Chem., 2020, vol. 90, no. 6, p. 948.

https://doi.org/10.1134/S1070363220060043

21. Kažoka, H., Krauze, A., Viļums, M., Černova, L., Sīle, L., and Duburs, G., Chem. Heterocycl. Compd., 2007, vol. 43, no. 1 , p. 50.

https://doi.org/10.1007/s10593-007-0007-z

22. Kalashnik, I.N. and Dyachenko, V.D., Russ. J. Gen. Chem., 2020, vol. 90, no. 3, p. 357.

https://doi.org/10.1134/S1070363220030068

23. Dyachenko, V.D., Matusov, I.O., Dyachenko, I.V., and Nenajdenko, V.G., Russ. J. Org. Chem., 2018, vol. 54, no. 12, p. 1777.

https://doi.org/10.1134/S1070428018120060

24. Dotsenko, V.V., Krivokolysko, S.G., Litvinov, V.P., and Chernega, A.N., Chem. Heterocycl. Compd., 2007, vol. 43 , no. 5 , p. 599.

https://doi.org/10.1007/s10593-007-0094-x

25. Krauze, A. and Duburs, G., Chem. Heterocycl. Compd., 1996, vol. 32, no. 8, p. 986.

https://doi.org/10.1007/BF01176979

26. Dyachenko, V.D., Russ. J. Org. Chem., 2007, vol. 43, no. 2, p. 271.

https://doi.org/10.1134/S1070428007020194

27. Shestopalov, A.M., Rodinovskaya, L.A., Sharanin, Yu.A., and Litvinov, V.P., J. Gen. Chem. USSR, 1988, vol. 58, no. 4, p. 745.

28. Rodinovskaya, L.A., Belukhina, E.V., Shestopalov, A.M., and Litvinov, V.P., Russ. Chem. Bull., 1994, vol. 43, no. 3 , p. 449.

https://doi.org/10.1007/BF01169725

29. Sharanin, Yu.A., Goncharenko, M.P., Shestopalov, A.M., Litvinov, V.P., and Turov, A.V., J. Org. Chem. USSR, 1991, vol. 27 , no. 9.2 , p. 1767.

30. Dotsenko, V.V., Krivokolysko, S.G., and Litvinov, V.P., Russ. Chem. Bull., 2012, vol. 61, no. 1, p. 131. https://doi.org/10.1007/s11172-012-0018-5

31. Frolov, K.A., Dotsenko, V.V., Krivokolysko, S.G., and Litvinov, V.P., Chem. Heterocycl. Compd., 2012, vol. 48, no. 7, p. 1117.

https://doi.org/10.1007/s10593-012-1109-9

32. Dotsenko, V.V., Frolov, K.A., and Krivokolysko, S.G., Chem. Heterocycl. Compd., 2015, vol. 51, no. 2, p. 109. https://doi.org/10.1007/s10593-015-1668-7

33. Dotsenko, V.V., Frolov, K.A., Chigorina, E.A., Khrustaleva, A.N., Bibik, E.Yu., and Krivokolysko, S.G., Russ. Chem. Bull., 2019, vol. 68, no. 4, p. 691. https://doi.org/10.1007/s11172-019-2476-5

34. Dotsenko, V.V., Chigorina, E.A., Papaianina, E.S., Frolov, K.A., and Krivokolysko, S.G., Macroheterocycles,
2015, vol. 8, no. 3 , p. 310 .

https://doi.org/10.6060/mhc150870d

35. Dotsenko, V.V., Suikov, S.Yu., Pekhtereva, T.M., and Krivokolysko, S.G., Chem. Heterocycl. Compd., 2013, vol. 49, no. 7, p. 1009.

https://doi.org/10.1007/s10593-013-1339-5

36. Dotsenko, V.V., Suikov, S.Yu., Pekhtereva, T.M., and Krivokolysko, S.G., Chem. Heterocycl. Compd., 2013, vol. 49, no. 7, p. 1009.

https://doi.org/10.1007/s10593-013-1339-5

37. Gewald, K., Schäfer, H., and Schlegel, U., J. Prakt. Chem., 1976, vol. 318, no. 5, p. 779.

https://doi.org/10.1002/prac.19763180509

38. Krauze, A.A., Bomika, Z.A., Pelcher, Y.É., Mazheika, I.B., and Dubur, G.Ya., Chem. Heterocycl. Compd., 1982, vol. 18, no. 4, p. 385.

https://doi.org/10.1007/BF00503559

39. Paronikyan, E.G., Noravyan, A.S., Dzhagatspanyan, I.A., Nazaryan, I.M., and Paronikyan, R.G., Pharm. Chem. J., 2002, vol. 36, no. 9, p. 465.

https://doi.org/10.1023/A:1021836504036

40. Moryashova, S.I, Salamandra, L.K., Fedorov, A.E., Rodinovskaya, L.A., Shestopalov, A.M., and Semenov, V.V., Russ. Chem. Bull., 1998, vol. 47, no. 2, p. 357. https://doi.org/10.1007/BF02498968

41. Osolodkin, D.I., Kozlovskaya, L.I., Dueva, E.V., Dotsenko, V.V., Rogova, Y.V., Frolov, K.A., Krivokolysko, S.G., Romanova, E.G., Morozov, A.S., Karganova, G.G., Palyulin, V.A., Pentkovski, V.M., and Zefirov, N.S., ACS Med. Chem. Lett., 2013, vol. 4, no. 9, p. 869.

https://doi.org/10.1021/ml400226s

42. Bibik, E.Yu., Yaroshevskaya, O.G., Devdera, A.V., Demenko, A.V., Zakharov, V.V., Frolov, K.A., Dotsenko, V.V., and Krivokolysko, S.G., Pharm. Chem. J., 2017, vol. 51, no. 8, p. 648 .

https://doi.org/10.1007/s11094-017-1669-1

43. Bibik, E.Yu., Saphonova, A.A., Yeryomin, A.V., Frolov, K.A., Dotsenko, V.V., and Krivokolysko, S.G., Res. Results Pharmacol., 2017, vol. 3, no. 4, p. 20. https://doi.org/10.18413/2313-8971-2017-3-4-20-25

44. Bibik, E.Yu., Nekrasa, I.A., Demenko, A.V., Frolov, K.A., Dotsenko, V.V., and Krivokolysko, S.G., Bull. Sib. Med., 2019, vol. 18, no. 3, p. 21. https://doi.org/10.20538/1682-0363-2019-3-21-28

45. Rice, W.G., Turpin, J.A., Schaeffer, C.A., Graham, L., Clanton, D., Buckheit, R.W., Zaharevitz, D., Summers, M.F., Wallqvist, A., and Covell, D.G., J. Med. Chem., 1996, vol. 39, no. 19, p. 3606.

https://doi.org/10.1021/jm960375o

46. Norman, D.D., Ibezim, A., Scott, W.E., White, S., Parrill, A.L., and Baker, D.L., Bioorg. Med. Chem., 2013, vol. 21, no. 17, p. 5548.

https://doi.org/10.1016/j.bmc.2013.05.061 
47. Mullan, M.J., Paris, D., and Bakshi, P., WO Patent 2008070875, 2008.

48. Sander, T., OSIRIS Property Explorer, Idorsia Pharmaceuticals Ltd., Switzerland. http://www.organic-chemistry.org/prog/peo/.

49. Daina, A., Michielin, O., and Zoete, V., Sci. Rep., 2017, vol. 7, Article N 42717. https://doi.org/10.1038/srep42717

50. Gfeller, D., Grosdidier, A., Wirth, M., Daina, A., Michielin, O., and Zoete, V., Nucl. Acids Res., 2014, vol. 42, no. W1, p. W32. https://doi.org/10.1093/nar/gku293

51. Molinspiration Property Calculation Service. Molinspiration Cheminformatics, Slovak Republic, 2002. https:/www.molinspiration.com/.

52. Cheng, F., Li, W., Zhou, Y., Shen, J., Wu, Z., Liu, G., Lee, P.W., and Tang, Y., J. Chem. Inf. Model., 2012, vol. 52, no. 11, p. 3099. https://doi.org/10.1021/ci300367a

53. Litvinov, V.P., Krivokolysko, S.G., and Rusanov, E.B., Doklady Chem., 2001, vol. 377, nos. 4-6, p. 94. https://doi.org/10.1023/A:1019205013304

54. Krivokolysko, S.G., Dyachenko, V.D., Chernega, A.N., and Litvinov, V.P., Russ. Chem. Bull., 2000, vol. 49, no. 4 , p. 736 . https://doi.org/10.1007/BF02495493

55. Krivokolysko, S.G., Dyachenko, V.D., Nesterov, V.N., Sharanin, Yu.A., and Struchkov, Yu.T., Russ. J. Org. Chem., 1999, vol. 35, no. 6, p. 942.

56. Krivokolysko, S.G., Dyachenko, V.D., and Litvinov, V.P., Russ. Chem. Bull., 2000, vol. 49, no. 3, p. 487. https://doi.org/10.1007/BF02494780

57. Ramabadran, K., Bansinath, M., Turndorf, H., and Puig, M.M., J. Pharm. Methods, 1989, Vol. 21, no. 1, p. 21. https://doi.org/10.1016/0160-5402(89)90019-3

58. Sewell, R.D.E. and Spencer, P.S.J., Neuropharmacology, 1976, vol. 15, no. 11, p. 683. https://doi.org/10.1016/0028-3908(76)90037-X

59. Kotlinska, J.H., Gibula-Bruzda, E., Witkowska, E., Chung, N.N., Schiller, P.W., and Izdebski, J., Peptides, 2013, vol. 39, p. 103.

https://doi.org/10.1016/j.peptides.2012.11.008
60. Hamura, H., Yoshida, M., Shimizu, K., Matsukura, T., Suzuki, H., Narita, M., and Suzuki, T., Jap. J. Pharmacol., 2000, vol. 83, no. 4, p. 286.

https://doi.org/10.1254/jjp.83.286

61. Raboisson, P. and Dallel, R., Neurosci. Biobehav. Rev., 2004, vol. 28, no. 2, p. 219.

https://doi.org/10.1016/j.neubiorev.2003.12.003

62. Pereira, P.J.S., Dornelles, F.N., Santos, D.S., Calixto, J.B., Morrone, F.B., and Campos, M.M., Int. Immunopharmacol., 2009, vol. 9, no. 1, p. 80. https://doi.org/10.1016/j.intimp.2008.10.001

63. Le Bars, D., Gozariu, M., and Cadden, S.W., Ann. Fr. Anesth. Reanim., 2001, vol. 20, no. 4, p. 347. https://doi.org/10.1016/S0750-7658(01)00381-1

64. Krzyzanowska, A. and Avendaño, C., Brain Behav., 2012, vol. 2 , no. 5 , p. 678 . https://doi.org/10.1002/brb3.85

65. Clavelou, P., Dallel, R., Orliaguet, T., Woda, A., and Raboisson, P., Pain, 1995, vol. 62, no. 3, p. 295. https://doi.org/10.1016/0304-3959(94)00273-H

66. Brunskill, J.S.A., De, A., and Ewing, D.F., J. Chem. Soc. Perkin Trans. 1, 1978, no. 6, p. 629. https://doi.org/10.1039/P19780000629

67. Nesterova, I.N., Shanazarov, A.K., Poznyak, A.M., Lakoza, M.I., Shemeryankin, B.V., and Granik, V.G., Pharm. Chem. J., 1994, vol. 28, no. 8, p. 583. https://doi.org/10.1007/BF02219035

68. Karkishchenko, N.N. and Grachev, S.V., Al'ternativy biomeditsiny. Rukovodstvo po laboratornym zhivotnym $i$ al'ternativnym modelyam v biomeditsinskikh tekhnologiyakh (Iomedicine Alternatives. Guide to Laboratory Animals and Alternative Models in Biomedical Technologies), Moscow: Profil', 2010, p. 26.

69. Khabriyev, R.U., Rukovodstvo po eksperimental'nomu (doklinicheskomu) izucheniyu novykh farmakologicheskikh sredstv [Guidelines for Experimental (Preclinical) Study of New Pharmacological Agents], Moscow: Meditsina, 2005, p. 338.

70. Kobzar', A.I., Prikladnaya matematicheskaya statistika (Applied Mathematical Statistics), Moscow: Fizmatlit, 2006, p. 454. 